\title{
APPENDIX I. PHYSICAL PROPERTIES DATA, DEEP SEA DRILLING PROJECT LEG 93, SITES 603, 604, AND 605 ${ }^{1}$
}

\author{
Dean A. Dunn, Department of Geology, University of Southern Mississippi \\ Brian N. M. Biart, Exploration Consultants Ltd. \\ and \\ Mark W. Johns, Department of Oceanography, Texas A\&M University ${ }^{2}$
}

On Leg 93, physical properties measurements were made of vertical and horizontal sonic velocity, acoustic impedance, vane shear strength, and penetrometer strength, using procedures discussed in Boyce $(1973,1976,1984)$. Gravimetric procedures were used to determine wet-bulk density, grain density, porosity, and water content, using either the chunk method (Boyce, 1973) or the cylinder method (Boyce, 1984). Calcium carbonate content of Leg 93 sediments was determined by the carbonate bomb (Müller and Gastner, 1971; Dunn, 1980).

\section{SONIC VELOCITY}

Compressional-wave velocities were measured on the Hamilton Frame Velocimeter by timing a $400-\mathrm{kHz}$ pulse between two transducers and by measuring the distance across the sample with a dial gauge. Velocities in unconsolidated sediments were generally measured on the split core. Correction factors established by Boyce (1976) were used to compensate for increased thickness and travel time $(0.242 \mathrm{~cm}$ and $1.108 \mu \mathrm{s}$, respectively) caused by the polycarbonate liner. For consolidated sediments, a piece was removed from the core and trimmed carefully to form two parallel surfaces to insure good contact with the transducer heads. Salt water was used to make an acoustic contact between the sample and the transducers. On indurated sediments, measurements were made of both horizontal (perpendicular to the core axis) and vertical (parallel to the core axis) sonic velocities. By measuring aluminum, Lucite, and brass standards of known lengths and assumed velocities, correction factors were determined for the oscilloscope.

Horizontal sonic velocities were determined for Hole 603 sediments (Table 1). Both horizontal and vertical velocity were measured in Hole 603B (Table 2) and Hole 605 (Table 5). Vertical velocities were determined for Hole 603C (Table 3) and Hole 604 (Table 4) sediments. Acoustic impedance of Leg 93 sediments was determined from the vertical sonic velocity, and impedance data are listed for Holes 603B (Table 2), 603C (Table 3), 604 (Table 4), and 605 (Table 5).

\footnotetext{
1 van Hinte, J. E., Wise, S. W., Jr., et al., Init. Repts. DSDP, 93: Washington (U.S. Govt, Printing Office).

2 Addresses: (Dunn) Department of Geology, University of Southern Mississippi, Southern Station Box 5044, Hattiesburg, MS 39406-5044; (Biart) Exploration Consultants Ltd., Highlands Farm, Greys Road, Henley-on-Thames, Oxon RG9 4PS, United Kingdom; (Johns, present address) Tetra Tech, Inc., 11820 Northup Way, Suite 100, Bellevue, WA 98005.
}

\section{VANE SHEAR AND PENETROMETER STRENGTH}

A Soiltest Torvane and a Soiltest CL-700 Pocket Penetrometer were used to determine the shear strength and the unconfined compression strength of Leg 93 sediments and sedimentary rocks. The Torvane blades were buried about $0.5 \mathrm{~cm}$ below the sediment surface of the split core halves, with the vane axis oriented parallel to the bedding plane of the sediment. The Torvane was hand-rotated at a rate designed to reach failure in about $10 \mathrm{~s}$ with constant loading. Measurements were made in the least-disturbed sections of the split core, parallel to the core axis, and were discontinued when cracking of the sediments was observed, indicating failure by fracturing rather than by shear. Vane shear data was determined for sediments in Cores 603-1 and -2 (Table 1), and for the entire recovered section of Hole $603 \mathrm{C}$ (Table 3 ) and Hole 604 (Table 4). Two blades on the Torvane fractured in the last sample from Hole 604, preventing the determination of shear strength values for Hole 605 samples.

The Pocket Penetrometer measured compression strength parallel to bedding. It was pushed into undisturbed sections of the split core, perpendicular to the split face of the core. Unconfined compression strength was determined for sediments from Hole 604 (Table 4) and for Cores 605-3 to -6 (Table 5).

\section{GRAVIMETRIC PROCEDURES}

Wet-bulk density, grain density, porosity, and water content were all determined for sample intervals at which sonic velocities were determined, generally at intervals of $1.5 \mathrm{~m}$ (one sample per core section). The cylinder technique (Boyce, 1984) was used on soft or stiff sediments. Samples were taken with stainless steel or brass cylinders of known volume and weight, and were tightly enclosed to retain moisture until they could be weighed. For indurated sediment and hard rock, the gravimetric chunk technique (Boyce, 1973) was used, in which samples cut out of the core for measurement of sonic velocities were stored under seawater. Chunk volume was determined by weighing both in air and suspended under water.

Sample volume and weight yield the wet-bulk density without a buoyancy correction. Wet-water content and porosity were calculated from the water loss after drying 
at $110^{\circ} \mathrm{C}$ for $24 \mathrm{hr}$. Water content is related to the weight of the wet samples and therefore is defined here as wetwater content. All wet-water content and porosity data were corrected for salt content, using the correction factors for seawater (Boyce, 1976). The approximate grain density was calculated from the gravimetric data. Porosity was calculated by assuming that the sediments were water-saturated. This may not be true for all samples, because some undergo adiabatic expansions if the in situ pressure is released when cores are brought from the seafloor to the shipboard laboratory. The effect of the volume increase of the samples due to elastic rebound, caused by the removal of in situ overburden pressure (Hamilton, 1976), was not taken into account.

\section{CARBONATE CONTENT}

Calcium carbonate percentages of Leg 93 sediments were determined by the "Karbonat-Bombe" procedure of Müller and Gastner (1971), modified to eliminate the gas vapor pressure effect of using concentrated hydrochloric acid in the bombs (Dunn, 1980). Dried samples of sediment and rock were ground, and approximately $1 \mathrm{~g}$ of sample was reacted with concentrated $\mathrm{HC} 1$ in a closed cylinder. The resulting increase in pressure was measured on a manometer gauge. Pressure increase caused by formation of $\mathrm{CO}_{2}$ gas is proportional to the concen- tration of $\mathrm{CaCO}_{3}$ in the sample, and carbonate percentages of samples were determined by analyzing standards of known carbonate content.

\section{REFERENCES}

Boyce, R. E., 1973. Appendix I. Physical property methods. In Edgar, N. T., Saunders, J. B., et al., Init. Repts. DSDP, 15: Washington (U.S. Govt. Printing Office), 1115-1128.

1976. Definitions and laboratory techniques of compressional sound velocity parameters and wet-water content, wet-bulk density, and porosity parameters by gravimetric and gamma ray attenuation techniques. In Schlanger, S. O., Jackson, E. D., et al., Init. Repts. DSDP, 33: Washington (U.S. Govt. Printing Office), 931-958.

1984. Leg 75 methods for laboratory measured physical properties, Gearhart-Owen well logs, and the Uyeda downhole temperature probe. In Hay, W. W., Sibuet, J.-C., et al., Init. Repts. DSDP , 75, Pt. 2: Washington (U.S. Govt. Printing Office), 11791187.

Dunn, D. A., 1980. Revised techniques for quantitative calcium carbonate analysis using the "Karbonat-Bombe," and comparisons to other quantitative carbonate analysis methods. J. Sed. Petrol., 50: 631-637.

Hamilton, E. L., 1976. Variations of density and porosity with depth in deep-sea sediments. J. Sed. Petrol., 46:280-300.

Müller, G., and Gastner, M., 1971. The "Karbonat-Bombe," a simple device for determination of the carbonate content in sediments, soils and other materials. $N$. Jb. Miner, Mh., 10:466-469.

Date of Acceptance: 2 July 1986 
Table 1. Summary of physical properties data for samples from Hole 603.

\begin{tabular}{|c|c|c|c|c|c|c|c|c|}
\hline $\begin{array}{c}\text { Core-Section } \\
\text { (interval in } \mathrm{cm} \text { ) }\end{array}$ & $\begin{array}{c}\text { Sub-bottom } \\
\text { depth }(\mathrm{m})\end{array}$ & $\begin{array}{c}\text { Horizontal } \\
\text { sonic velocity } \\
(\mathrm{km} / \mathrm{s})\end{array}$ & $\begin{array}{l}\text { Shear } \\
\text { strength } \\
(\mathrm{kPa})\end{array}$ & $\begin{array}{c}\text { Bulk } \\
\text { density } \\
\left(\mathrm{g} / \mathrm{cm}^{3}\right)\end{array}$ & $\begin{array}{c}\text { Grain } \\
\text { density } \\
\left(\mathrm{g} / \mathrm{cm}^{3}\right)\end{array}$ & $\begin{array}{c}\text { Porosity } \\
(\%)\end{array}$ & $\begin{array}{c}\text { Water } \\
\text { content } \\
(\%)\end{array}$ & $\begin{array}{c}\text { Carbonate } \\
\text { content } \\
(\%)\end{array}$ \\
\hline $1-1,20$ & 0.20 & & 15.80 & & & & & \\
\hline $1-1,20$ & 0.20 & & 26.71 & & & & & \\
\hline $1-1,70$ & 0.70 & & 23.71 & & & & & 19 \\
\hline $1-1,120$ & 1.20 & & 23.45 & & & & & \\
\hline $1-2,30$ & 1.80 & & 24.01 & & & & & \\
\hline $1-2,80$ & 2.30 & & 23.36 & & & & & \\
\hline $1-2,130$ & 2.80 & & 23.53 & & & & & \\
\hline $1-3,90$ & 3.90 & & 27.00 & & & & & \\
\hline $1-4,30$ & 4.80 & & 26.96 & & & & & \\
\hline $1-4,130$ & 5.80 & & 26.82 & & & & & \\
\hline $1-5,80$ & 6.80 & & 26.95 & & & & & \\
\hline $1-6,80$ & 8.30 & & & 1.47 & 2.60 & 72 & 96 & 17 \\
\hline $2-1,90$ & 9.90 & & 17.62 & & & & & \\
\hline $2-3,83$ & 12.83 & & & 1.69 & 2.72 & 61 & 57 & 11 \\
\hline $2-5,32$ & 15.32 & & & 1.67 & 2.71 & 61 & 59 & 6 \\
\hline $3-1,17$ & 83.97 & & & 1.65 & 2.63 & 61 & 60 & 14 \\
\hline $4-1,80$ & 123.00 & & & 1.65 & 2.53 & 59 & 56 & 8 \\
\hline $4-1,93$ & 123.13 & 1.517 & & & & & & \\
\hline $4-2,80$ & 124.50 & 1.296 & & & & & & \\
\hline $4-2,80$ & 124.50 & 1.296 & & & & & & \\
\hline $4-2,80$ & 124.50 & 1.546 & & & & & & \\
\hline $4-2,80$ & 124.50 & & & 1.68 & 2.64 & 59 & 56 & 15 \\
\hline $4-3,40$ & 125.60 & 1.502 & & & & & & \\
\hline $4-3,80$ & 126.00 & & & 1.67 & 2.74 & 63 & 61 & 10 \\
\hline $4-3,99$ & 126.19 & 1.553 & & & & & & \\
\hline $4-3,148$ & 126.68 & 1.466 & & & & & & \\
\hline $4.4,23$ & 126.93 & 1.437 & & & & & & \\
\hline $4.4,23$ & 126.93 & 1.499 & & & & & & \\
\hline $4.4,80$ & 127.50 & & & 1.74 & 3.35 & 69 & 68 & 6 \\
\hline 4.4 .98 & 127.68 & 1.474 & & & & & & \\
\hline $4-4,142$ & 128.12 & 1.505 & & & & & & \\
\hline $4-5,23$ & 128.43 & 1.499 & & & & & & \\
\hline $4-5,80$ & 129.00 & & & 1.62 & 2.60 & 62 & 64 & 14 \\
\hline $4-5,128$ & 129.48 & 1.500 & & & & & & \\
\hline $4-6,70$ & 130.40 & & & 1.58 & 2.57 & 64 & 70 & 7 \\
\hline $5-1,84$ & 132.64 & & & 1.59 & 2.49 & 62 & 65 & i \\
\hline $5-2,80$ & 134.10 & & & 1.70 & 2.57 & 57 & 51 & 13 \\
\hline $5-3,80$ & 135.60 & & & 1.74 & 2.64 & 56 & 49 & 12 \\
\hline $5-4,80$ & 137.10 & & & 1.62 & 2.42 & 57 & 56 & 18 \\
\hline $7 \cdot 1,80$ & 180.60 & & & 1.67 & 2.57 & 58 & 54 & \\
\hline $7-2,80$ & 182.10 & & & 1.65 & 2.31 & 51 & 46 & \\
\hline $7-3,80$ & 183.60 & & & 1.70 & 2.56 & 56 & 50 & \\
\hline $7-4,80$ & 185.10 & & & 1.64 & 2.58 & 61 & 60 & 8 \\
\hline $7-5,80$ & 186.60 & & & 1.65 & 2.57 & 59 & 57 & 3 \\
\hline $7-6,80$ & 188.10 & & & 1.66 & 2.57 & 59 & 55 & 3 \\
\hline $8-1,80$ & 199.80 & & & 1.74 & 2.62 & 55 & 47 & 13 \\
\hline $8-2,60$ & 201.10 & & & 1.74 & 2.62 & 55 & 47 & 19 \\
\hline $8-3,45$ & 202.45 & & & 1.53 & 2.27 & 59 & 64 & 10 \\
\hline $9-1,80$ & 209.40 & & & 1.69 & 2.45 & 54 & 47 & 15 \\
\hline $9-1,130$ & 209.90 & 1.538 & & & & & & \\
\hline $9-2,80$ & 210.90 & & & 1.72 & 2.50 & 53 & 45 & 6 \\
\hline $9-2,130$ & 211.40 & 1.585 & & & & & & \\
\hline $9-3,80$ & 212.40 & 1.525 & & & & & & \\
\hline $9-3,80$ & 212.40 & & & 1.77 & 2.55 & 51 & 42 & 8 \\
\hline $9-4,80$ & 213.90 & & & 1.69 & 2.58 & 57 & 52 & 19 \\
\hline $9-4,117$ & 214.27 & 1.518 & & & & & & \\
\hline $9-4,117$ & 214.27 & 1.548 & & & & & & \\
\hline $10-1,80$ & 219.00 & & & & & & & \\
\hline $10-2,80$ & 220.50 & & & 1.78 & 2.62 & 53 & 43 & 10 \\
\hline $10-2,130$ & 221.00 & 1.548 & & & & & & \\
\hline $10-3,80$ & 222.00 & & & 1.80 & 2.66 & 53 & 42 & 10 \\
\hline $10-4,80$ & 223.50 & & & 1.79 & 2.65 & 53 & 43 & 1 \\
\hline $10-4,81$ & 223.51 & 1.241 & & & & & & \\
\hline $10-5,80$ & 225.00 & & & 1.78 & 2.58 & 51 & 41 & 4 \\
\hline $10-6,72$ & 226.42 & 1.453 & & & & & & \\
\hline $11-1,80$ & 228.60 & & & 1.61 & 2.46 & 60 & 60 & 9 \\
\hline $11-2,35$ & 229.65 & & & 1.73 & 2.55 & 54 & 46 & 7 \\
\hline $11-3,80$ & 231.60 & & & 1.71 & 2.51 & 54 & 47 & \\
\hline $11-4,80$ & 233.10 & & & 1.62 & 2.54 & 61 & 60 & 7 \\
\hline $11-5,80$ & 234.60 & & & 1.77 & 2.80 & 58 & 50 & 6 \\
\hline $11-6,32$ & 235.62 & & & 1.69 & 2.63 & 59 & 54 & 7 \\
\hline $12-1,80$ & 267.00 & & & 1.69 & 2.52 & 55 & 49 & \\
\hline $12-2,80$ & 268.50 & & & 1.80 & 2.65 & 53 & 42 & 2 \\
\hline $12-2,105$ & 268.75 & 1.395 & & & & & & \\
\hline $12-3,60$ & 269.80 & 1.524 & & & & & & \\
\hline $12-3,80$ & 270.00 & & & 1.76 & 2.51 & 50 & 41 & 2 \\
\hline $12, \mathrm{CC}$ & 270.50 & 1.877 & & & & & & \\
\hline
\end{tabular}


Table 1 (continued).

\begin{tabular}{|c|c|c|c|c|c|c|c|c|}
\hline $\begin{array}{c}\text { Core-Section } \\
\text { (interval in cm) }\end{array}$ & $\begin{array}{c}\begin{array}{c}\text { Sub-bottom } \\
\text { depth }(\mathrm{m})\end{array} \\
\end{array}$ & $\begin{array}{c}\text { Horizontal } \\
\text { sonic velocity } \\
(\mathrm{km} / \mathrm{s})\end{array}$ & $\begin{array}{l}\text { Shear } \\
\text { strength } \\
(\mathrm{kPa})\end{array}$ & $\begin{array}{c}\text { Bulk } \\
\text { density } \\
\left(\mathrm{g} / \mathrm{cm}^{3}\right)\end{array}$ & $\begin{array}{l}\text { Grain } \\
\text { density } \\
\left(\mathrm{g} / \mathrm{cm}^{3}\right)\end{array}$ & $\begin{array}{c}\text { Porosity } \\
(\%)\end{array}$ & $\begin{array}{c}\text { Water } \\
\text { content } \\
(\%)\end{array}$ & $\begin{array}{c}\text { Carbonate } \\
\text { content } \\
(\%)\end{array}$ \\
\hline $13-1,80$ & 276.60 & & & 1.81 & 2.64 & 52 & 41 & 7 \\
\hline $13-2,80$ & 278.10 & & & 1.78 & 2.53 & 50 & 40 & 9 \\
\hline $13-3,80$ & 279.60 & & & 1.81 & 2.64 & 52 & 41 & 17 \\
\hline $13-4,80$ & 281.10 & & & 1.73 & 2.36 & 47 & 38 & \\
\hline $13-5,80$ & 282.60 & & & 1.64 & 2.18 & 47 & 41 & 11 \\
\hline $13-6,80$ & 284.10 & & & 1.80 & 2.60 & 51 & 41 & 5 \\
\hline $14-1,79$ & 314.99 & & & 1.61 & 2.32 & 55 & 52 & 10 \\
\hline $14-2,72$ & 316.42 & 1.468 & & & & & & \\
\hline $14-2,80$ & 316.50 & & & 1.71 & 2.57 & 56 & 50 & 11 \\
\hline $14-3,79$ & 317.99 & & & 1.68 & 2.33 & 50 & 42 & 8 \\
\hline $14-3,81$ & 318.01 & 1.550 & & & & & & \\
\hline $14-4,7$ & 318.77 & 1.538 & & & & & & \\
\hline $14-4,79$ & 319.49 & & & 1.68 & 2.30 & 49 & 42 & 11 \\
\hline $14-5,11$ & 320.31 & & & 1.80 & 2.61 & 51 & 41 & 13 \\
\hline $15-1,74$ & 324.54 & 1.154 & & & & & & \\
\hline $15-1,78$ & 324.58 & & & 1.75 & 2.67 & 56 & 48 & 16 \\
\hline $15-2,52$ & 325.82 & 1.577 & & & & & & \\
\hline $15-2,78$ & 326.08 & & & & & & & 1 \\
\hline $15-3,78$ & 327.58 & & & 1.65 & 2.48 & 57 & 54 & 1 \\
\hline $15-4,78$ & 329.08 & & & 1.73 & 2.57 & 54 & 47 & 6 \\
\hline $15-4,100$ & 329.30 & 1.551 & & & & & & \\
\hline $15-5,6$ & 329.86 & 1.560 & & & & & & \\
\hline $15-5,78$ & 330.58 & & & 1.75 & 2.68 & 56 & 48 & 1 \\
\hline $15-6,78$ & 332.08 & & & 1.66 & 2.56 & 58 & 55 & 2 \\
\hline $16-1,69$ & 362.89 & 1.564 & & & & & & \\
\hline $16-1,80$ & 363.00 & & & 1.74 & 2.37 & 47 & 38 & 17 \\
\hline $16-2,69$ & 364.39 & 1.363 & & & & & & \\
\hline $16-2,80$ & 364.50 & & & 1.70 & 2.32 & 48 & 40 & 18 \\
\hline $16-3,80$ & 366.00 & & & 1.79 & 2.52 & 49 & 38 & 1 \\
\hline $16-4,40$ & 367.10 & 1.557 & & & & & & \\
\hline $16-4,80$ & 367.50 & & & 1.78 & 2.48 & 48 & 38 & 4 \\
\hline $17-1,10$ & 371.90 & & & 1.75 & 2.50 & 51 & 41 & 6 \\
\hline $17-1,14$ & 371.94 & 1.679 & & & & & & \\
\hline $17-2,52$ & 373.82 & 1.592 & & & & & & \\
\hline $17-2,55$ & 373.85 & & & 1.80 & 2.54 & 49 & 38 & \\
\hline $17-5,14$ & 377.94 & 1.655 & & & & & & \\
\hline $17-5,15$ & 377.95 & & & 1.80 & 2.52 & 48 & 37 & 5 \\
\hline $18-1,14$ & 410.34 & & & 1.75 & 2.40 & 48 & 38 & \\
\hline $18-1,93$ & 411.13 & 1.678 & & & & & & \\
\hline $18-1,93$ & 411.13 & 1.640 & & & & & & \\
\hline $19-1,79$ & 420.59 & 1.114 & & & & & & \\
\hline $19-1,80$ & 420.60 & & & 1.69 & 2.32 & 48 & 41 & 6 \\
\hline $19-3,79$ & 423.59 & 1.183 & & & & & & \\
\hline $19-3,80$ & 423.60 & & & 1.79 & 2.57 & 50 & 39 & 4 \\
\hline $19-5,80$ & 426.60 & & & 1.82 & 2.53 & 48 & 36 & \\
\hline $20-1,80$ & 449.40 & & & 1.75 & 2.51 & 51 & 42 & \\
\hline $20-2,52$ & 450.62 & 1.476 & & & & & & \\
\hline $20-2,80$ & 450.90 & & & 1.81 & 2.46 & 46 & 34 & \\
\hline $20-3,50$ & 452.10 & 1.517 & & & & & & \\
\hline $20-3,80$ & 452.40 & & & 1.74 & 2.22 & 40 & 31 & \\
\hline $20-4,80$ & 453.90 & 1.598 & & & & & & \\
\hline $20-4,80$ & 453.90 & & & $\begin{array}{l}1.81 \\
1.85\end{array}$ & $\begin{array}{l}2.48 \\
2.50\end{array}$ & $\begin{array}{l}46 \\
44\end{array}$ & $\begin{array}{l}34 \\
31\end{array}$ & 1 \\
\hline $20-5,20$ & 454.80 & & & $\begin{array}{l}1.85 \\
1.70\end{array}$ & $\begin{array}{l}2.50 \\
2.18\end{array}$ & $\begin{array}{l}44 \\
42\end{array}$ & $\begin{array}{l}31 \\
34\end{array}$ & $\frac{1}{2}$ \\
\hline $\begin{array}{l}21-1,79 \\
21-1,80\end{array}$ & $\begin{array}{l}458.99 \\
459.00\end{array}$ & 1.448 & & & 2.18 & 42 & 34 & \\
\hline $\begin{array}{l}21-1,80 \\
21-1,80\end{array}$ & $\begin{array}{l}459.00 \\
459.00\end{array}$ & $\begin{array}{l}1.448 \\
1.489\end{array}$ & & & & & & \\
\hline $21-2,76$ & 460.46 & & & 1.88 & 2.52 & 43 & 30 & \\
\hline $21-2,80$ & 460.50 & 1.597 & & & & & & \\
\hline $21-3,80$ & 462.00 & & & 1.87 & 2.51 & 44 & 33 & \\
\hline $21-3,80$ & 462.00 & 1.814 & & & & & & \\
\hline $21-4,32$ & 463.02 & 1.546 & & & & & & \\
\hline $22-1,80$ & 468.60 & & & 1.82 & 2.38 & 43 & 33 & 3 \\
\hline $22-2,80$ & 470.10 & 1.411 & & & & & & \\
\hline $22-2,80$ & 470.10 & & & 1.83 & 2.50 & 47 & 37 & \\
\hline $22-3,88$ & 471.68 & & & 1.86 & 2.53 & 46 & 36 & \\
\hline $22-3,88$ & 471.68 & 1.591 & & & & & & \\
\hline $22-4,84$ & 473.14 & 1.572 & & & & & & \\
\hline $\begin{array}{l}22-4,84 \\
22-5,80\end{array}$ & $\begin{array}{l}473.14 \\
474.60\end{array}$ & & & 1.88 & 2.57 & 46 & 35 & 3 \\
\hline $22-5,80$ & 474.60 & 1.521 & & & & & & \\
\hline $22-6,79$ & 476.09 & & & 1.90 & 2.61 & 46 & 34 & \\
\hline $22-6,79$ & 476.09 & 1.261 & & & & & & \\
\hline $23-1,82$ & 507.02 & 1.525 & & 183 & 252 & 47 & 38 & \\
\hline $\begin{array}{l}23-1,82 \\
23-2,83\end{array}$ & $\begin{array}{l}507.02 \\
508.53\end{array}$ & 1.573 & & 1.85 & 2.32 & 41 & 38 & \\
\hline $23-2,83$ & 508.53 & & & 1.87 & 2.57 & 47 & 36 & \\
\hline $23-3,89$ & 510.09 & 1.608 & & & & & & \\
\hline
\end{tabular}


Table 1 (continued).

\begin{tabular}{|c|c|c|c|c|c|c|c|c|}
\hline $\begin{array}{l}\text { Core-Section } \\
\text { (interval in } \mathrm{cm} \text { ) }\end{array}$ & $\begin{array}{l}\text { Sub-bottom } \\
\text { depth }(\mathrm{m})\end{array}$ & $\begin{array}{l}\text { Horizontal } \\
\text { sonic velocity } \\
(\mathrm{km} / \mathrm{s})\end{array}$ & $\begin{array}{l}\text { Shear } \\
\text { strength } \\
(\mathrm{kPa})\end{array}$ & $\begin{array}{c}\text { Bulk } \\
\text { density } \\
\left(\mathrm{g} / \mathrm{cm}^{3}\right)\end{array}$ & $\begin{array}{c}\text { Grain } \\
\text { density } \\
\left(\mathrm{g} / \mathrm{cm}^{3}\right)\end{array}$ & $\begin{array}{c}\text { Porosity } \\
(\%)\end{array}$ & $\begin{array}{c}\text { Water } \\
\text { content } \\
(\%)\end{array}$ & $\begin{array}{c}\text { Carbonate } \\
\text { content } \\
(\%)\end{array}$ \\
\hline $23-3,89$ & 510.09 & & & 1.77 & 2.36 & 45 & 37 & \\
\hline $23-4,84$ & 511.54 & & & 1.85 & 2.55 & 47 & 37 & 5 \\
\hline $23-4,84$ & 511.54 & 1.520 & & & & & & \\
\hline $23-5,20$ & 512.40 & 1.170 & & & & & & \\
\hline $23-5,20$ & 512.40 & & & 1.86 & 2.57 & 47 & 37 & 4 \\
\hline $24-1,80$ & 516.60 & 1.577 & & & & & & \\
\hline $24-1,80$ & 516.60 & & & 1.79 & 2.43 & 46 & 38 & 3 \\
\hline $24-2,81$ & 518.11 & 1.540 & & & & & & \\
\hline $24-2,84$ & 518.14 & & & 2.02 & 2.98 & 50 & 36 & \\
\hline $24-3,81$ & 519.61 & & & 1.89 & 2.71 & 50 & 39 & 2 \\
\hline $24-3,81$ & 519.61 & 1.549 & & & & & & \\
\hline $24-4,80$ & 521.10 & 1.498 & & & & & & \\
\hline $24-4,80$ & 521.10 & & & 1.85 & 2.58 & 48 & 38 & 1 \\
\hline $24-5,80$ & 522.60 & 1.476 & & & & & & \\
\hline $24-5,80$ & 522.60 & & & 1.88 & 2.65 & 48 & 38 & \\
\hline $24-6,81$ & 524.11 & 1.536 & & & & & & \\
\hline $25-1,40$ & 545.00 & & & 1.84 & 2.81 & 56 & 47 & 2 \\
\hline $25-1,60$ & 545.20 & 1.575 & & & & & & \\
\hline $25-2,60$ & 546.70 & & & 1.82 & 2.63 & 51 & 43 & \\
\hline $25-2,80$ & 546.90 & 1.513 & & & & & & \\
\hline $25-3,84$ & 548.44 & 1.464 & & & & & & \\
\hline $25-3,84$ & 548.44 & & & 1.64 & 2.15 & 47 & 44 & \\
\hline $25-4,83$ & 549.93 & 1.554 & & & & & & \\
\hline $25-4,83$ & 549.93 & & & 1.82 & 2.54 & 49 & 40 & 3 \\
\hline $25-5,6$ & 550.66 & & & $\begin{array}{l}1.02 \\
1.82\end{array}$ & 2.62 & 51 & 43 & \\
\hline $25-5,85$ & 551.45 & 1.487 & & & & & & \\
\hline $25-6,80$ & 552.90 & 1.538 & & & & & & \\
\hline $25-6,80$ & 552.90 & & & 1.84 & 2.61 & 49 & 40 & 2 \\
\hline $25-7,33$ & 553.93 & 1.567 & & & & & & \\
\hline $25, \mathrm{CC}$ & 554.40 & & & 1.86 & 2.61 & 49 & 38 & 3 \\
\hline $26-1,90$ & 555.10 & 1.447 & & & & & & \\
\hline $26-1,130$ & 555.50 & & & 1.85 & 2.57 & 48 & 38 & \\
\hline $26-2,92$ & 556.62 & 1.708 & & & & & & \\
\hline $26-2,130$ & 557.00 & & & 1.87 & 2.70 & 51 & 40 & \\
\hline $26-3,95$ & 558.15 & 1.760 & & & & & & \\
\hline $26-3,130$ & 558.50 & & & 1.85 & 2.63 & 50 & 40 & \\
\hline $26-4,94$ & 559.64 & 1.570 & & & & & & \\
\hline $26-4,130$ & 560.00 & & & 1.82 & 2.57 & 50 & 41 & 2 \\
\hline $26-5,96$ & 561.16 & 1.524 & & & & & & \\
\hline $26-5,130$ & 561.50 & & & 1.84 & 2.60 & 49 & 39 & \\
\hline $26-6,65$ & 562.35 & & & 1.84 & 2.62 & 50 & 41 & \\
\hline $26-6,66$ & 562.36 & 1.372 & & & & & & \\
\hline $27, \mathrm{CC}$ & 564.00 & 1.722 & & & & & & \\
\hline $27, \mathrm{CC}$ & 564.00 & & & 1.78 & 2.38 & 45 & 37 & 2 \\
\hline $28-1,33$ & 563.33 & 1.509 & & 1.83 & 2.45 & 45 & 35 & 5 \\
\hline $29-1,53$ & 573.93 & 1.646 & & & & & & \\
\hline $29-1,135$ & 574.75 & & & 1.85 & 2.54 & 47 & 37 & 3 \\
\hline $29-2,53$ & 575,43 & 1.725 & & & & & & \\
\hline $29-2,135$ & 576.25 & & & 1.89 & 2.68 & 49 & 37 & 2 \\
\hline $29-3,51$ & 576.91 & 1.737 & & & & & & \\
\hline $29-3,135$ & 577.75 & & & 1.88 & 2.62 & 47 & 36 & \\
\hline $29-4,51$ & 578.41 & 1.587 & & & & & & \\
\hline $29-4,135$ & 579.25 & & & 1.87 & 2.60 & 47 & 37 & 4 \\
\hline $29-5,115$ & 580.55 & 1.564 & & & & & & \\
\hline $29-5,135$ & 580.75 & & & 1.88 & 2.68 & 50 & 39 & 2 \\
\hline $30-1,98$ & 583.98 & & & 1.86 & 2.79 & 54 & 44 & i \\
\hline $30-1,139$ & 584.39 & 1.539 & & & & & & \\
\hline $30-2,77$ & 585.27 & & & 1.86 & 2.67 & 50 & 70 & \\
\hline $30-2,81$ & 585.31 & 1.471 & & & & & & \\
\hline $30-3,77$ & 586.77 & & & 1.87 & 2.61 & 48 & 37 & \\
\hline $30-3,81$ & 586.81 & 1.666 & & & & & & \\
\hline $30-4,77$ & 588.27 & & & 1.89 & 2.60 & 46 & 35 & \\
\hline $30-4,81$ & 588.31 & 1.598 & & & & & & \\
\hline $30-5,12$ & 589.12 & & & 1.89 & 2.64 & 48 & 37 & 1 \\
\hline $30-5,17$ & 589.17 & 1.471 & & & & & & \\
\hline $31-1,71$ & 593.31 & 1.674 & & 1.88 & 2.68 & 49 & 38 & 2 \\
\hline $31-2,119$ & 595.29 & & & $\begin{array}{l}1.00 \\
1.89\end{array}$ & 2.61 & 46 & 35 & 2 \\
\hline $31-2,121$ & 595.31 & 1.791 & & & & & & \\
\hline $31-3,71$ & 596.31 & & & 1.89 & 2.57 & 45 & 34 & 2 \\
\hline $31-3,72$ & 596.32 & 1.404 & & & & & & \\
\hline $32-1,55$ & 602.75 & 1.746 & & & & & & \\
\hline $32-1,58$ & 602.78 & & & 1.91 & 2.61 & 45 & 34 & 1 \\
\hline $32-2,58$ & 604.28 & & & 1.90 & 2.62 & 46 & 35 & \\
\hline $32-2,94$ & 604.64 & 1.702 & & & & & & \\
\hline $33-1,69$ & 612.49 & 1.669 & & & & & & \\
\hline $33-1,70$ & 612.50 & & & 1.90 & 2.60 & 46 & 35 & \\
\hline $33-2,70$ & 614.00 & 1.784 & & & & & & \\
\hline
\end{tabular}


Table 1 (continued).

\begin{tabular}{|c|c|c|c|c|c|c|c|c|}
\hline $\begin{array}{l}\text { Core-Section } \\
\text { (interval in cm) }\end{array}$ & $\begin{array}{c}\text { Sub-bottom } \\
\text { depth }(\mathrm{m})\end{array}$ & $\begin{array}{c}\text { Horizontal } \\
\text { sonic velocity } \\
(\mathrm{km} / \mathrm{s})\end{array}$ & $\begin{array}{l}\text { Shear } \\
\text { strength } \\
(\mathrm{kPa})\end{array}$ & $\begin{array}{c}\text { Bulk } \\
\text { density } \\
\left(\mathrm{g} / \mathrm{cm}^{3}\right)\end{array}$ & $\begin{array}{c}\text { Grain } \\
\text { density } \\
\left(\mathrm{g} / \mathrm{cm}^{3}\right)\end{array}$ & $\begin{array}{c}\text { Porosity } \\
(\%)\end{array}$ & $\begin{array}{c}\text { Water } \\
\text { content } \\
(\%)\end{array}$ & $\begin{array}{c}\text { Carbonate } \\
\text { content } \\
(\%)\end{array}$ \\
\hline $33-2,70$ & 614.00 & & & 1.91 & 2.70 & 48 & 37 & \\
\hline $33-2,70$ & 614.00 & 1.722 & & & & & & \\
\hline $33-3,66$ & 615.46 & 1.687 & & & & & & \\
\hline $33-3,67$ & 615.47 & 1.651 & & & & & & \\
\hline $\begin{array}{l}33-3,67 \\
33-3,67\end{array}$ & $\begin{array}{l}615.47 \\
615.47\end{array}$ & 1.660 & & 1.92 & 2.60 & 44 & 32 & 2 \\
\hline $\begin{array}{l}33-3,07 \\
33-3,69\end{array}$ & $\begin{array}{l}013.41 \\
615.49\end{array}$ & 1.707 & & 1.92 & 2.00 & 44 & 32 & 2 \\
\hline $33-4,67$ & 616.97 & & & 1.89 & 2.59 & 46 & 35 & \\
\hline $33-5,75$ & 618.55 & & & 1.92 & 2.57 & 43 & 32 & \\
\hline $33-6,28$ & 619.58 & & & 1.95 & 2.68 & 45 & 33 & 6 \\
\hline $34-1,81$ & 622.21 & 1.573 & & 1.94 & 2.66 & 45 & 33 & 2 \\
\hline $34-2,76$ & 623.66 & 1.525 & & 1.94 & 2.68 & 45 & 33 & 2 \\
\hline $34-3,80$ & 625.20 & 1.710 & & 1.95 & 2.75 & 47 & 35 & 2 \\
\hline $34-4,77$ & 626.67 & 1.652 & & 1.68 & 2.11 & 41 & 34 & 6 \\
\hline $35-1,79$ & 631.79 & 1.641 & & 1.93 & 2.70 & 47 & 35 & \\
\hline $35-2,80$ & 633.30 & 1.707 & & 1.92 & 2.65 & 46 & 34 & \\
\hline $35-3,81$ & 634.81 & 1.676 & & 1.96 & 2.73 & 46 & 34 & 2 \\
\hline $35-4,77$ & 636.27 & 1.653 & & 1.95 & 2.74 & 47 & 34 & 6 \\
\hline $35-5,77$ & 637.77 & 1.594 & & 1.92 & 2.77 & 50 & 38 & 5 \\
\hline $35-6,75$ & 639.25 & 1.659 & & 1.95 & 2.69 & 45 & 33 & \\
\hline $36-1,73$ & 641.33 & & & 1.95 & 2.93 & 52 & 40 & \\
\hline $36-1,77$ & 641.37 & 1.628 & & 1.93 & 2.67 & 46 & 34 & 13 \\
\hline $36-1,81$ & 641.41 & 1.610 & & & & & & \\
\hline $36-2,80$ & 642.90 & & & 1.92 & 2.76 & 49 & 37 & 1 \\
\hline $36-2,81$ & 642.91 & 1.615 & & 1.95 & 2.65 & 44 & 32 & \\
\hline $36-2,81$ & 642.91 & 1.511 & & & & & & \\
\hline $36-3,73$ & 644.33 & 1.603 & & 1.90 & 2.70 & 49 & 38 & 12 \\
\hline $36-3,77$ & 644.37 & 1.665 & & & & & & \\
\hline $36-4,75$ & 645.85 & 1.606 & & 1.95 & 2.68 & 45 & 33 & 2 \\
\hline $\begin{array}{l}36-4,83 \\
37-1,66\end{array}$ & $\begin{array}{l}645.93 \\
650.86\end{array}$ & $\begin{array}{l}1.651 \\
1.587\end{array}$ & & 1.92 & 2.73 & 48 & 36 & \\
\hline $37-2,55$ & 652.25 & 1.693 & & 1.95 & 2.73 & $\begin{array}{l}40 \\
47\end{array}$ & 34 & 6 \\
\hline $37-3,15$ & 653.35 & 1.635 & & 1.95 & 2.67 & 45 & 32 & \\
\hline $38-1,80$ & 660.60 & 1.558 & & & & & & \\
\hline $38-1,80$ & 660.60 & 1.615 & & 1.93 & 2.66 & 46 & 34 & \\
\hline $\begin{array}{l}38-1,80 \\
38-2,80\end{array}$ & $\begin{array}{l}660.60 \\
662.10\end{array}$ & 1.607 & & & & & & \\
\hline $38-2,80$ & 662.10 & & & 1.94 & 2.65 & 45 & 33 & 3 \\
\hline $38-3,14$ & 662.94 & 1.624 & & & & & & \\
\hline $38-3,14$ & 662.94 & & & 1.93 & 2.68 & 46 & 34 & 2 \\
\hline $39-1,70$ & 670.10 & 1.678 & & 1.93 & 2.66 & 46 & 33 & 3 \\
\hline $39-2,51$ & 671.41 & 1.581 & & 1.93 & 2.81 & 51 & 39 & \\
\hline $40-1,81$ & 679.81 & 1.692 & & 1.92 & 2.70 & 47 & 36 & \\
\hline $40-2,79$ & 681.29 & 1.660 & & 1.95 & 2.66 & 44 & 32 & \\
\hline $40-3,75$ & 682.75 & 1.642 & & 1.90 & 2.69 & 49 & 37 & \\
\hline $40-4,79$ & 684.29 & 1.539 & & 1.91 & 2.77 & 50 & 39 & 4 \\
\hline $40-5,82$ & 685.82 & 1.650 & & 1.92 & 2.74 & 49 & 37 & 3 \\
\hline $40-6,80$ & 687.30 & 1.670 & & 1.92 & 2.71 & 48 & 36 & 1 \\
\hline $41-1,80$ & 689.40 & 1.780 & & 1.93 & 2.64 & 45 & 32 & 3 \\
\hline $41-2,80$ & 690.90 & 1.809 & & 1.93 & 2.69 & 47 & 34 & 3 \\
\hline $41-3,80$ & 692.40 & & & 1.92 & 2.80 & 51 & 39 & 6 \\
\hline $41-4,80$ & 693.90 & 1.809 & & 1.90 & 2.67 & 48 & 37 & 6 \\
\hline $41-5,80$ & 695.40 & 1.679 & & 1.91 & 2.67 & 47 & 36 & 5 \\
\hline $41-6,80$ & 696.90 & 1.766 & & 1.88 & 2.63 & 48 & 37 & 2 \\
\hline $42-1,74$ & 698.94 & 1.731 & & 1.86 & 2.66 & 50 & 70 & 5 \\
\hline $42-2,74$ & 700.44 & 1.702 & & 1.87 & 2.73 & 51 & 41 & 4 \\
\hline $42-3,74$ & 701.94 & 1.671 & & 1.82 & 2.62 & 41 & 43 & 4 \\
\hline $42-4,74$ & 703.44 & 1.710 & & 1.85 & 2.66 & 51 & 41 & 4 \\
\hline $43-1,72$ & 708.52 & 1.775 & & & & & & \\
\hline $43-1,72$ & 708.52 & 1.876 & & & & & & \\
\hline $43-1,74$ & 708.54 & & & 1.89 & 2.69 & 49 & 38 & 4 \\
\hline $44-1,55$ & 717.95 & 1.734 & & 1.88 & 2.62 & 48 & 37 & 2 \\
\hline $44-2,55$ & 719.45 & 1.727 & & 1.85 & 2.56 & 47 & 37 & 3 \\
\hline $44-3,55$ & 720.95 & 1.783 & & 1.84 & 2.59 & 49 & 39 & 3 \\
\hline $44-4,55$ & 722.45 & 1.802 & & 1.91 & 2.61 & 45 & 33 & 2 \\
\hline $45-1,70$ & 727.70 & 1.807 & & 1.88 & 2.64 & 48 & 37 & 3 \\
\hline $45-2,70$ & 729.20 & 1.791 & & 1.88 & 2.62 & 47 & 36 & 3 \\
\hline $45-3,70$ & 730.70 & 1.732 & & 1.87 & 2.70 & 50 & 39 & \\
\hline $45-4,70$ & 732.20 & 1.745 & & 1.87 & 2.64 & 48 & 38 & 2 \\
\hline $45-5,69$ & 733.69 & 1.715 & & 1.88 & 2.63 & 48 & 37 & 3 \\
\hline $46-1,80$ & 737.40 & 1.640 & & 1.78 & 2.54 & 51 & 44 & 6 \\
\hline $46-2,90$ & 739.00 & 1.681 & & 1.80 & 2.49 & 48 & 40 & 3 \\
\hline $46-3,80$ & 740.40 & 1.655 & & 1.75 & 2.50 & 52 & 46 & \\
\hline $46-4,77$ & 741.87 & 1.722 & & & & & & \\
\hline $46-4,78$ & 741.88 & 1.608 & & 1.83 & 2.49 & 46 & 36 & 3 \\
\hline $46, \mathrm{CC}$ & 742.65 & 1.720 & & 1.89 & 2.56 & 45 & 33 & 10 \\
\hline $47-1,70$ & 746.90 & 1.597 & & 1.88 & 2.53 & 44 & 34 & 4 \\
\hline
\end{tabular}


Table 1 (continued).

\begin{tabular}{|c|c|c|c|c|c|c|c|c|}
\hline $\begin{array}{c}\text { Core-Section } \\
\text { (interval in cm) }\end{array}$ & $\begin{array}{l}\text { Sub-bottom } \\
\text { depth }(\mathrm{m})\end{array}$ & $\begin{array}{c}\text { Horizontal } \\
\text { sonic velocity } \\
(\mathrm{km} / \mathrm{s})\end{array}$ & $\begin{array}{l}\text { Shear } \\
\text { strength } \\
(\mathrm{kPa})\end{array}$ & $\begin{array}{c}\text { Bulk } \\
\text { density } \\
\left(\mathrm{g} / \mathrm{cm}^{3}\right)\end{array}$ & $\begin{array}{l}\text { Grain } \\
\text { density } \\
\left(\mathrm{g} / \mathrm{cm}^{3}\right)\end{array}$ & $\begin{array}{c}\text { Porosity } \\
(\%)\end{array}$ & $\begin{array}{c}\text { Water } \\
\text { content } \\
(\%)\end{array}$ & $\begin{array}{c}\text { Carbonate } \\
\text { content } \\
(\%)\end{array}$ \\
\hline $47, \mathrm{CC}$ & 748.17 & 1.818 & & 2.00 & 2.52 & 36 & 23 & \\
\hline $48-1,80$ & 756.60 & 1.830 & & & & & & 3 \\
\hline $49-1,80$ & 766.20 & 1.806 & & 1.86 & 2.51 & 45 & 35 & 6 \\
\hline $49-2,80$ & 767.70 & 1.797 & & 1.85 & 2.45 & 43 & 32 & 4 \\
\hline $49-3,58$ & 768.98 & 1.717 & & & & & & \\
\hline $49-3,58$ & 768.98 & 1.799 & & 1.89 & 2.52 & 44 & 33 & 4 \\
\hline $50-1,76$ & 775.76 & 1.811 & & 1.85 & 2.51 & 46 & 36 & 4 \\
\hline $50-2,82$ & 777.32 & 1.768 & & 1.80 & 2.51 & 49 & 41 & 4 \\
\hline $50-3,82$ & 778.82 & 1.757 & & 1.79 & 2.47 & 48 & 40 & 3 \\
\hline $50-4,86$ & 780.36 & 1.799 & & 1.78 & 2.50 & 50 & 42 & 2 \\
\hline $51-1,48$ & 785.08 & 1.527 & & 1.91 & 2.57 & 43 & 32 & 3 \\
\hline $51-1,48$ & 785.08 & 1.671 & & & & & & \\
\hline $51-2,48$ & 786.58 & 1.671 & & 1.86 & 2.50 & 44 & 33 & 3 \\
\hline $52-1,80$ & 795.00 & & & 1.81 & 2.49 & 48 & 39 & \\
\hline $52-1,80$ & 795.00 & 1.662 & & & & & & \\
\hline $52-2,80$ & 796.50 & & & 1.84 & 2.52 & 47 & 37 & 4 \\
\hline $52-3,80$ & 798.00 & 1.729 & & 1.90 & 2.51 & 42 & 31 & 7 \\
\hline $52-4,80$ & 799.50 & 1.678 & & 1.91 & 2.60 & 45 & 33 & \\
\hline $52-5,10$ & 800.30 & 1.614 & & 1.90 & 2.58 & 45 & 33 & 4 \\
\hline $53-1,41$ & 804.21 & 1.496 & & 1.91 & 2.56 & 44 & 32 & 2 \\
\hline $54-1,74$ & 823.74 & 1.751 & & & & & & \\
\hline
\end{tabular}


Table 2. Summary of physical properties data for samples from Hole 603B.




Table 2 (continued).

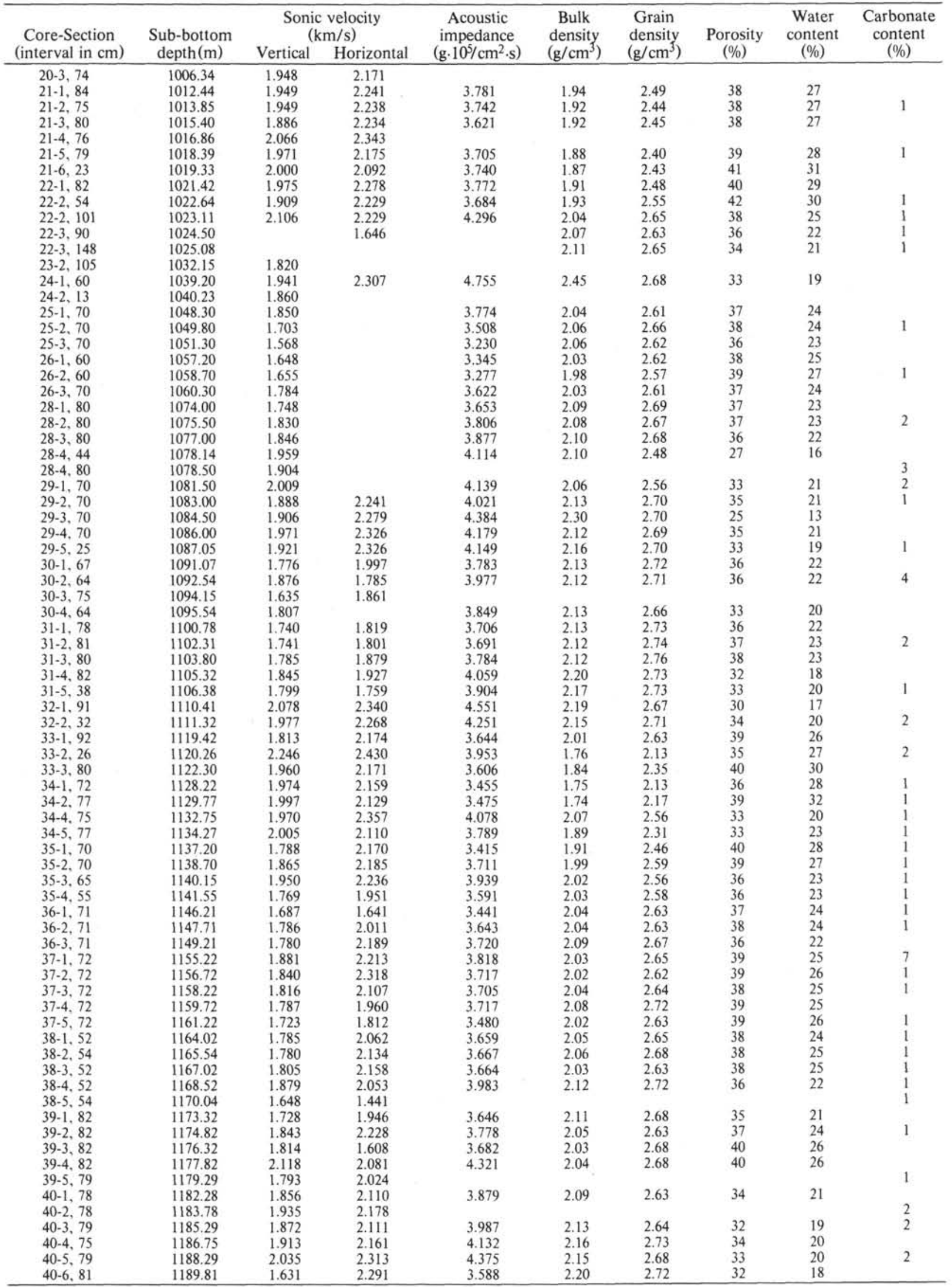


Table 2 (continued).

\begin{tabular}{|c|c|c|c|c|c|c|c|c|c|}
\hline $\begin{array}{c}\text { Core-Section } \\
\text { (interval in cm) }\end{array}$ & $\begin{array}{l}\text { Sub-bottom } \\
\text { depth }(\mathrm{m})\end{array}$ & \multicolumn{2}{|c|}{$\begin{array}{c}\text { Sonic velocity } \\
(\mathrm{km} / \mathrm{s})\end{array}$} & $\begin{array}{c}\text { Acoustic } \\
\text { impedance } \\
\left(\mathrm{g} \cdot 10^{5} / \mathrm{cm}^{2} \cdot \mathrm{s}\right)\end{array}$ & $\begin{array}{l}\text { Bulk } \\
\text { density } \\
\left(\mathrm{g} / \mathrm{cm}^{3}\right)\end{array}$ & $\begin{array}{l}\text { Grain } \\
\text { density } \\
\left(\mathrm{g} / \mathrm{cm}^{3}\right)\end{array}$ & $\begin{array}{c}\text { Porosity } \\
(\%)\end{array}$ & $\begin{array}{c}\text { Water } \\
\text { content } \\
(\%)\end{array}$ & $\begin{array}{c}\text { Carbonate } \\
\text { content } \\
(\%)\end{array}$ \\
\hline $41-1,77$ & 1191.27 & 1.974 & 2.200 & 4.284 & 2.17 & 2.70 & 32 & 19 & \\
\hline $41-2,79$ & 1192.79 & 1.955 & 2.239 & 4.242 & 2.17 & 2.73 & 33 & 20 & \\
\hline $41-3,87$ & 1194.37 & 1.946 & 2.223 & 4.242 & 2.18 & 2.72 & 33 & 19 & \\
\hline $41-4,84$ & 1195.84 & 2.084 & 2.270 & 4.564 & 2.19 & 2.73 & 32 & 19 & \\
\hline $42-1,90$ & 1198.10 & 2.055 & 2.110 & 4.439 & 2.16 & 2.71 & 33 & 20 & \\
\hline $42-2,80$ & 1199.50 & 1.905 & 2.026 & 3.753 & 1.97 & 2.58 & 40 & 28 & 1 \\
\hline $42-3,77$ & 1200.97 & 1.857 & 2.094 & 3.751 & 2.02 & 2.63 & 39 & 25 & $i$ \\
\hline $42-4,82$ & 1202.52 & 1.820 & 2.201 & 3.676 & 2.02 & 2.62 & 39 & 26 & \\
\hline $42-5,85$ & 1204.05 & 1.925 & 2.188 & 3.985 & 2.07 & 2.64 & 36 & 23 & 2 \\
\hline $42-6,35$ & 1205.05 & 1.892 & 2.099 & 3.879 & 2.05 & 2.68 & 39 & 26 & \\
\hline $43-1,82$ & 1205.62 & 2.036 & 2.281 & 4.337 & 2.13 & & & & \\
\hline $43-2,81$ & 1207.11 & 2.043 & 2.211 & 4.454 & 2.18 & 2.75 & 33 & 19 & \\
\hline $43-3,82$ & 1208.62 & 2.027 & 2.310 & 4.419 & 2.18 & 2.74 & 33 & 19 & \\
\hline $43-4,82$ & 1210.12 & 1.954 & 2.118 & 4.162 & 2.13 & 2.69 & 34 & 20 & \\
\hline $43-5,78$ & 1211.58 & 1.912 & 2.129 & 4.168 & 2.18 & 2.73 & 33 & 19 & \\
\hline $43-6,20$ & 1212.50 & 1.843 & 1.980 & 4.257 & 2.31 & 2.69 & 24 & 12 & \\
\hline $44-1,77$ & 1215.17 & 2.006 & 2.292 & 4.172 & 2.08 & 2.60 & 34 & 21 & 15 \\
\hline $44-2,78$ & 1216.68 & 1.969 & 2.200 & 4.115 & 2.09 & 2.59 & 33 & 20 & 13 \\
\hline $44-3,77$ & 1218.17 & 1.925 & 2.217 & 4.043 & 2.10 & 2.66 & 35 & 22 & 3 \\
\hline $44-4,81$ & 1219.71 & 1.823 & 2.263 & 3.828 & 2.10 & 2.66 & 35 & 22 & 13 \\
\hline $45-1,71$ & 1224.71 & 1.735 & & 3.644 & 2.10 & 2.63 & 34 & 21 & 4 \\
\hline $49-1,141$ & 1263.81 & 1.879 & 2.040 & 3.946 & 2.10 & 2.65 & 34 & 21 & 9 \\
\hline $49-2,74$ & 1264.64 & 1.775 & 2.128 & 3.532 & 1.99 & 2.59 & 40 & 27 & 40 \\
\hline $49-3,72$ & 1266.12 & 1.936 & 2.252 & 4.124 & 2.13 & 2.67 & 33 & 20 & 32 \\
\hline $49-4,72$ & 1267.62 & 1.882 & 2.069 & 3.726 & 1.98 & 2.60 & 40 & 27 & 40 \\
\hline $49-5,34$ & 1268.74 & 1.953 & 2.218 & 3.926 & 2.01 & 2.56 & 37 & 24 & 78 \\
\hline $50-1,70$ & 1272.70 & 2.159 & 2.165 & 4.620 & 2.14 & 2.64 & 32 & 19 & 30 \\
\hline $51-1,73$ & 1282.33 & 2.128 & 2.333 & 4.703 & 2.21 & & & & \\
\hline $51-2,88$ & 1283.98 & 2.138 & 2.519 & 4.447 & 2.08 & 2.62 & 35 & 22 & 90 \\
\hline $51-3,73$ & 1285.33 & 1.994 & 2.433 & 4.227 & 2.12 & 2.62 & 32 & 19 & 8 \\
\hline $51-4,90$ & 1287.00 & 2.348 & 2.655 & 5.025 & 2.14 & 2.62 & 31 & 18 & 84 \\
\hline $51-5,82$ & 1288.42 & 2.022 & 2.254 & 4.287 & 2.12 & 2.65 & 33 & 20 & 39 \\
\hline $51-6,93$ & 1290.03 & 2.155 & 2.840 & 4.612 & 2.14 & & & & 82 \\
\hline $52-1,79$ & 1291.99 & 1.890 & 2.459 & 3.912 & 2.07 & 2.60 & 35 & 22 & 76 \\
\hline $52-2,78$ & 1293.48 & 2.053 & 2.188 & 4.168 & 2.03 & 2.54 & 34 & 22 & 76 \\
\hline $52-3,75$ & 1294.95 & 2.057 & 2.067 & 4.258 & 2.07 & 2.59 & 34 & 21 & 21 \\
\hline $52-4,81$ & 1296.51 & 1.889 & 2.217 & 3.910 & 2.07 & & & & 21 \\
\hline $52-5,75$ & 1297.95 & 2.055 & 2.146 & 4.398 & 2.14 & 2.65 & 32 & 19 & 15 \\
\hline $52-6,85$ & 1299.55 & 1.902 & 2.188 & 3.899 & 2.05 & 2.62 & 37 & 23 & 13 \\
\hline $53-1,73$ & 1301.53 & 1.941 & 1.734 & 4.076 & 2.10 & 2.65 & 34 & 21 & 23 \\
\hline $53-2,77$ & 1303.07 & 1.904 & 2.154 & 3.941 & 2.07 & 2.65 & 37 & 23 & 16 \\
\hline $53-3,76$ & 1304.56 & 1.948 & 2.156 & 4.071 & 2.09 & 2.62 & 34 & 21 & 22 \\
\hline $53-4,79$ & 1306.09 & 1.870 & 2.101 & 3.721 & 1.99 & 2.56 & 38 & 26 & 66 \\
\hline $53-5,76$ & 1307.56 & 2.380 & 2.474 & 5.212 & 2.19 & 2.65 & 29 & 16 & 79 \\
\hline $53-6,40$ & 1308.70 & 1.919 & 2.177 & 4.049 & 2.11 & 2.63 & 33 & 20 & 44 \\
\hline $54-1,79$ & 1311.19 & 2.078 & 2.067 & 4.509 & 2.17 & 2.60 & 28 & 16 & 4 \\
\hline $54-2,89$ & 1312.79 & 1.918 & 2.215 & 4.047 & 2.11 & 2.62 & 33 & 20 & 34 \\
\hline $54-3,92$ & 1314.32 & 2.049 & 1.983 & 4.344 & 2.12 & 2.62 & 32 & 19 & 6 \\
\hline $54-4,85$ & 1315.75 & 2.056 & 2.069 & 4.420 & 2.15 & 2.63 & 31 & 18 & 3 \\
\hline $54-5,71$ & 1317.11 & 1.969 & 2.133 & 4.096 & 2.08 & 2.62 & 35 & 21 & 25 \\
\hline $55-1,70$ & 1320.70 & 1.608 & 2.045 & 3.393 & 2.11 & 2.67 & 35 & 21 & 21 \\
\hline $55-2,72$ & 1322.22 & 1.633 & 1.832 & 3.462 & 2.12 & 2.62 & 32 & 19 & 3 \\
\hline $55-3,71$ & 1323.71 & 4.395 & 4.143 & 11.427 & 2.60 & 2.70 & 6 & 3 & 35 \\
\hline $55-4,77$ & 1325.27 & 1.845 & 2.216 & 4.004 & 2.17 & 2.71 & 33 & 19 & 5 \\
\hline $55-5,92$ & 1326.92 & 1.767 & 1.997 & 3.463 & 1.96 & 2.60 & 42 & 30 & 61 \\
\hline $56-1,72$ & 1330.32 & 2.065 & 2.077 & 4.522 & 2.19 & 2.65 & 29 & 17 & 5 \\
\hline $56-2,97$ & 1332.07 & 1.787 & 2.042 & 3.663 & 2.05 & 2.67 & 38 & 25 & 37 \\
\hline $56-3,97$ & 1333.57 & 1.828 & 2.135 & 3.839 & 2.10 & 2.64 & 34 & 21 & 52 \\
\hline $57-1,66$ & 1339.86 & 1.873 & 2.337 & 3.952 & 2.11 & 2.63 & 33 & 20 & 5 \\
\hline $57-2,70$ & 1341.40 & 1.991 & 2.207 & 4.042 & 2.03 & 2.59 & 37 & 24 & 58 \\
\hline $57-3,69$ & 1342.89 & 2.060 & 2.000 & 4.388 & 2.13 & 2.68 & 34 & 20 & 26 \\
\hline $57-4,69$ & 1344.39 & 3.994 & 4.166 & 10.265 & 2.57 & 2.73 & 10 & 4 & 27 \\
\hline $57-5,46$ & 1345.66 & 1.927 & 1.977 & 4.220 & 2.19 & 2.64 & 29 & 16 & 39 \\
\hline $57-6,67$ & 1347.37 & 1.941 & 1.784 & 4.231 & 2.18 & 2.69 & 32 & 18 & 3 \\
\hline $58-1,77$ & 1349.57 & 2.234 & 2.347 & 4.848 & 2.17 & 2.66 & 30 & 18 & 75 \\
\hline $58-2,77$ & 1351.07 & 1.927 & 1.934 & 3.950 & 2.05 & 2.56 & 34 & 22 & 3 \\
\hline $58-3,80$ & 1352.60 & 2.036 & 2.319 & 4.377 & 2.15 & 2.68 & 33 & 20 & 71 \\
\hline $58-4,80$ & 1354.10 & 2.061 & 2.061 & 4.328 & 2.10 & 2.60 & 33 & 20 & 5 \\
\hline $58-5,93$ & 1355.73 & 2.305 & 2.640 & 4.956 & 2.15 & 2.68 & 33 & 19 & 79 \\
\hline $58-6,37$ & 1356.67 & 2.103 & 2.518 & 4.395 & 2.09 & 2.59 & 33 & 20 & 82 \\
\hline $59-1,115$ & 1359.55 & 3.564 & 3.903 & 9.017 & 2.53 & 2.66 & 9 & 4 & 27 \\
\hline $59-2,116$ & 1361.06 & 1.930 & 2.360 & 4.207 & - 2.18 & 2.67 & 30 & 17 & 30 \\
\hline $59-3,128$ & 1362.68 & 2.722 & 2.609 & 5.607 & 2.06 & 2.58 & 34 & 21 & 79 \\
\hline $59-4,56$ & 1363.46 & 1.953 & 2.290 & 4.062 & 2.08 & 2.56 & 32 & 20 & 79 \\
\hline $59-5,58$ & 1364.98 & 1.970 & 2.106 & 4.236 & 2.15 & 2.61 & 30 & 17 & 6 \\
\hline $60-1,71$ & 1368.71 & 2.037 & 1.953 & 4.339 & 2.13 & 2.62 & 31 & 18 & 2 \\
\hline $60-2,70$ & 1370.20 & 2.072 & 2.145 & 4.517 & 2.18 & 2.66 & $\tau 30$ & 17 & 4 \\
\hline
\end{tabular}


Table 2 (continued).

\begin{tabular}{|c|c|c|c|c|c|c|c|c|c|}
\hline $\begin{array}{l}\text { Core-Section } \\
\text { (interval in } \mathrm{cm} \text { ) }\end{array}$ & $\begin{array}{c}\text { Sub-bottom } \\
\text { depth(m) }\end{array}$ & \multicolumn{2}{|c|}{$\begin{array}{l}\text { Sonic velocity } \\
(\mathrm{km} / \mathrm{s})\end{array}$} & $\begin{array}{c}\text { Acoustic } \\
\text { impedance } \\
\left(\mathrm{g} \cdot 10^{5} / \mathrm{cm}^{2} \cdot \mathrm{s}\right)\end{array}$ & $\begin{array}{c}\text { Bulk } \\
\text { density } \\
\left(\mathrm{g} / \mathrm{cm}^{3}\right)\end{array}$ & $\begin{array}{l}\text { Grain } \\
\text { density } \\
\left(\mathrm{g} / \mathrm{cm}^{3}\right)\end{array}$ & $\begin{array}{c}\text { Porosity } \\
(\%)\end{array}$ & $\begin{array}{c}\text { Water } \\
\text { content } \\
(\%)\end{array}$ & $\begin{array}{c}\text { Carbonate } \\
\text { content } \\
(\%)\end{array}$ \\
\hline $60-3,42$ & 1371.42 & 2.418 & 2.598 & 5.295 & 2.19 & 2.67 & 30 & 17 & 75 \\
\hline $60-4,42$ & 1372.92 & 2.001 & 2.134 & 4.342 & 2.17 & 2.66 & 30 & 17 & 13 \\
\hline $61-1,78$ & 1378.38 & & & & 2.65 & 2.73 & 5 & 2 & 40 \\
\hline $61-2,89$ & 1379.99 & 2.125 & 2.095 & 4.548 & 2.14 & 2.66 & 33 & 19 & 7 \\
\hline $61-3,71$ & 1381.31 & 2.331 & 2.651 & 5.082 & 2.18 & 2.66 & 30 & 17 & 74 \\
\hline $61-4,101$ & 1383.11 & 2.169 & 2.571 & 4.555 & 2.10 & 2.60 & 33 & 20 & 78 \\
\hline $61-5,87$ & 1384.47 & 1.908 & 2.109 & 3.969 & 2.08 & 2.66 & 36 & 23 & 19 \\
\hline $61-6,74$ & 1385.84 & 2.024 & 2.153 & 4.412 & 2.18 & 2.70 & 32 & 18 & 18 \\
\hline $62-1,79$ & 1387.99 & 2.109 & 2.173 & 4.619 & 2.19 & 2.70 & 31 & 18 & 56 \\
\hline $62-2,96$ & 1389.66 & 2.137 & 2.555 & 4.488 & 2.10 & 2.62 & 33 & 20 & 71 \\
\hline $62-3,100$ & 1391.20 & 2.021 & 2.066 & 4.244 & 2.10 & 2.65 & 35 & 21 & 10 \\
\hline $62-4,86$ & 1392.56 & 1.991 & 2.308 & 4.161 & 2.09 & 2.66 & 35 & 22 & 66 \\
\hline $62-5,83$ & 1394.03 & 2.603 & 2.582 & 5.883 & 2.26 & 2.69 & 27 & 14 & 79 \\
\hline $63-1,87$ & 1397.07 & 2.086 & 2.357 & 4.506 & 2.16 & 2.66 & 31 & 18 & 70 \\
\hline $63-2,85$ & 1398.55 & 2.334 & 2.568 & 5.158 & 2.21 & 2.70 & 30 & 17 & 77 \\
\hline $63-3,85$ & 1400.05 & 2.405 & 2.667 & 5.363 & 2.23 & 2.68 & 28 & 15 & 80 \\
\hline $63-4,74$ & 1401.44 & 2.181 & 2.289 & 4.733 & 2.17 & 2.64 & 30 & 17 & 66 \\
\hline $63-5,77$ & 1402.97 & 2.329 & 2.144 & 4.937 & 2.12 & 2.69 & 35 & 21 & 58 \\
\hline $63-6,80$ & 1404.50 & 2.284 & 2.612 & 4.979 & 2.18 & 2.63 & 29 & 16 & 77 \\
\hline $63-6,134$ & 1405.04 & 2.111 & 2.126 & 4.518 & 2.14 & 2.62 & 31 & 18 & 4 \\
\hline $64-1,76$ & 1406.46 & 2.044 & 2.102 & 4.456 & 2.18 & 2.62 & 28 & 16 & 12 \\
\hline $64-2,79$ & 1407.99 & 2.015 & 2.506 & 4.272 & 2.12 & 2.64 & 33 & 20 & 78 \\
\hline $64-3,75$ & 1409.45 & 2.015 & 2.307 & 4.171 & 2.07 & 2.57 & 33 & 20 & 75 \\
\hline $64-4,85$ & 1411.05 & 1.888 & 2.339 & 3.889 & 2.06 & 2.65 & 37 & 23 & 9 \\
\hline $64-5,75$ & 1412.45 & 2.274 & 2.426 & 5.048 & 2.22 & 2.64 & 27 & 15 & 81 \\
\hline $65-1,69$ & 1415.39 & 2.015 & 2.092 & 4.131 & 2.05 & 2.58 & 35 & 22 & 55 \\
\hline $65-2,81$ & 1417.01 & 2.210 & 2.290 & 4.729 & 2.14 & 2.61 & 30 & 18 & 4 \\
\hline $65-3,67$ & 1418.37 & 2.061 & 2.300 & 4.452 & 2.16 & 2.65 & 31 & 18 & 27 \\
\hline $65-4,70$ & 1419.90 & 2.263 & 2.576 & 4.865 & 2.15 & 2.62 & 31 & 18 & 75 \\
\hline $65-5,77$ & 1421.47 & 3.222 & 3.237 & & & & & & 84 \\
\hline $66-1,83$ & 1424.53 & 2.225 & 2.515 & 4.917 & 2.21 & 2.67 & 29 & 16 & 74 \\
\hline $66-2,93$ & 1426.13 & 1.971 & 2.341 & 4.119 & 2.09 & 2.64 & 35 & 22 & 3 \\
\hline $66-3,73$ & 1427.43 & 1.772 & 2.281 & 3.686 & 2.08 & 2.62 & 35 & 22 & 17 \\
\hline $66-4,81$ & 1429.01 & 2.448 & 2.862 & 5.435 & 2.22 & 2.67 & 28 & 16 & 72 \\
\hline $66-5,75$ & 1430.45 & 2.028 & 2.716 & 4.380 & 2.16 & 2.65 & 31 & 18 & 84 \\
\hline $67-1,68$ & 1433.38 & 2.094 & 2.562 & 4.397 & 2.10 & 2.62 & 33 & 20 & 78 \\
\hline $67-2,82$ & 1435.02 & 2.342 & 2.589 & 5.082 & 2.17 & 2.63 & 30 & 17 & 82 \\
\hline $67-3,71$ & 1436.41 & 2.271 & 2.039 & 4.746 & 2.09 & 2.63 & 34 & 21 & 4 \\
\hline $67-4,62$ & 1437.82 & 2.315 & 2.479 & 4.954 & 2.14 & 2.66 & 32 & 19 & 80 \\
\hline $67-5,76$ & 1439.46 & 1.866 & 2.400 & 4.012 & 2.15 & 2.66 & 32 & 19 & 18 \\
\hline $68-1,73$ & 1442.43 & 1.913 & 2.313 & 3.941 & 2.06 & 2.59 & 35 & 22 & 82 \\
\hline $68-2,73$ & 1443.93 & 1.999 & 2.116 & 4.358 & 2.18 & 2.63 & 33 & 20 & 6 \\
\hline $68-3,72$ & 1445.42 & 3.936 & 2.346 & 8.266 & 2.10 & 2.60 & 33 & 20 & 8 \\
\hline $68-4,97$ & 1447.17 & 2.577 & 1.643 & 5.618 & 2.18 & 2.63 & 29 & 17 & 85 \\
\hline $68-5,52$ & 1448.22 & 1.896 & 2.242 & 3.982 & 2.10 & 2.62 & 33 & 20 & 17 \\
\hline $69-1,90$ & 1451.60 & 1.879 & 2.333 & 3.983 & 2.12 & 2.65 & 33 & 20 & 12 \\
\hline $69-2,72$ & 1452.92 & 2.214 & 2.562 & 4.827 & 2.18 & 2.59 & 27 & 15 & 76 \\
\hline $69-3,94$ & 1454.64 & 3.583 & 2.411 & 6.378 & 1.78 & 2.14 & 33 & 25 & 76 \\
\hline $69-4,79$ & 1455.99 & 2.084 & 2.374 & 4.293 & 2.06 & 2.63 & 36 & 23 & 80 \\
\hline $69-5,70$ & 1457.40 & 2.112 & 2.546 & 4.499 & 2.13 & 2.66 & 33 & 20 & 79 \\
\hline $70-1,86$ & 1460.56 & 1.959 & 2.425 & 4.173 & 2.13 & 2.65 & 33 & 19 & 15 \\
\hline $70-2,83$ & 1462.03 & 2.320 & 2.580 & 4.965 & 2.14 & 2.68 & 33 & 20 & 77 \\
\hline $70-3,85$ & 1463.55 & 2.157 & 2.479 & 4.638 & 2.15 & 2.67 & 32 & 19 & 70 \\
\hline $70-4,82$ & 1465.02 & 2.262 & 2.509 & 4.818 & 2.13 & 2.66 & 33 & 20 & 80 \\
\hline $70-5,83$ & 1466.53 & 1.995 & 2.859 & 4.329 & 2.17 & 2.65 & 30 & 17 & 22 \\
\hline $71-1,80$ & 1469.50 & 2.135 & 2.146 & 4.548 & 2.13 & 2.54 & 28 & 16 & 4 \\
\hline $71-2,83$ & 1471.03 & 2.045 & 2.489 & 4.479 & 2.19 & 2.64 & 28 & 16 & 25 \\
\hline $71-3,81$ & 1472.51 & 2.130 & 2.268 & 4.665 & 2.19 & 2.59 & 26 & 15 & 11 \\
\hline $71-4,85$ & 1474.05 & 2.106 & 2.542 & 4.317 & 2.05 & 2.58 & 35 & 22 & 81 \\
\hline $71-5,80$ & 1475.50 & 2.330 & 2.540 & 4.940 & 2.12 & 2.57 & 30 & 18 & 77 \\
\hline $71-6,80$ & 1477.00 & 2.403 & 2.616 & 5.166 & 2.15 & 2.58 & 28 & 16 & 73 \\
\hline $73-1,72$ & 1483.42 & 2.007 & 2.440 & 4.315 & 2.15 & 2.62 & 30 & 18 & 18 \\
\hline $73-2,80$ & 1485.00 & 2.114 & 2.359 & 4.714 & 2.23 & 2.67 & 27 & 15 & 14 \\
\hline $73-3,76$ & 1486.46 & 2.691 & 2.521 & 5.866 & 2.18 & 2.70 & 32 & 18 & 68 \\
\hline $73-4,78$ & 1487.98 & 3.636 & 3.829 & 8.981 & 2.47 & 2.69 & 13 & 6 & 28 \\
\hline $73-5,65$ & 1489.35 & 1.974 & 2.378 & 4.126 & 2.09 & 2.74 & 39 & 25 & 77 \\
\hline $74-1,72$ & 1493.02 & 2.376 & 2.624 & 5.132 & 2.16 & 2.65 & 14 & 18 & 66 \\
\hline $74-2,70$ & 1494.50 & 2.456 & 2.879 & 5.428 & 2.21 & 2.71 & 30 & 17 & 91 \\
\hline $74-3,69$ & 1495.99 & 2.029 & 2.325 & 4.200 & 2.07 & 2.62 & 35 & 22 & 70 \\
\hline $74-4,70$ & 1497.50 & 2.355 & 2.316 & 5.181 & 2.20 & 2.71 & 31 & 17 & 77 \\
\hline $74-5,69$ & 1498.99 & 1.949 & 2.189 & 4.229 & 2.17 & 2.69 & 32 & 18 & 10 \\
\hline $74-6,70$ & 1500.50 & 2.132 & 2.436 & 4.648 & 2.18 & 2.69 & 31 & 18 & 68 \\
\hline $75-1,72$ & 1502.62 & 2.414 & 2.807 & 5.407 & 2.24 & 2.71 & 29 & 16 & \\
\hline $75-2,72$ & 1504.12 & 1.942 & 2.516 & 4.253 & 2.19 & 2.81 & 36 & 21 & 28 \\
\hline $75-3,66$ & 1505.56 & 2.074 & 2.457 & 4.521 & 2.18 & 2.65 & 32 & 19 & \\
\hline $75-4,42$ & 1506.82 & 2.158 & 2.600 & 4.575 & 2.12 & 2.64 & 33 & 20 & \\
\hline $75-5,42$ & 1508.32 & 1.927 & 2.353 & 4.047 & 2.10 & 2.71 & 37 & 23 & 18 \\
\hline
\end{tabular}


Table 2 (continued).

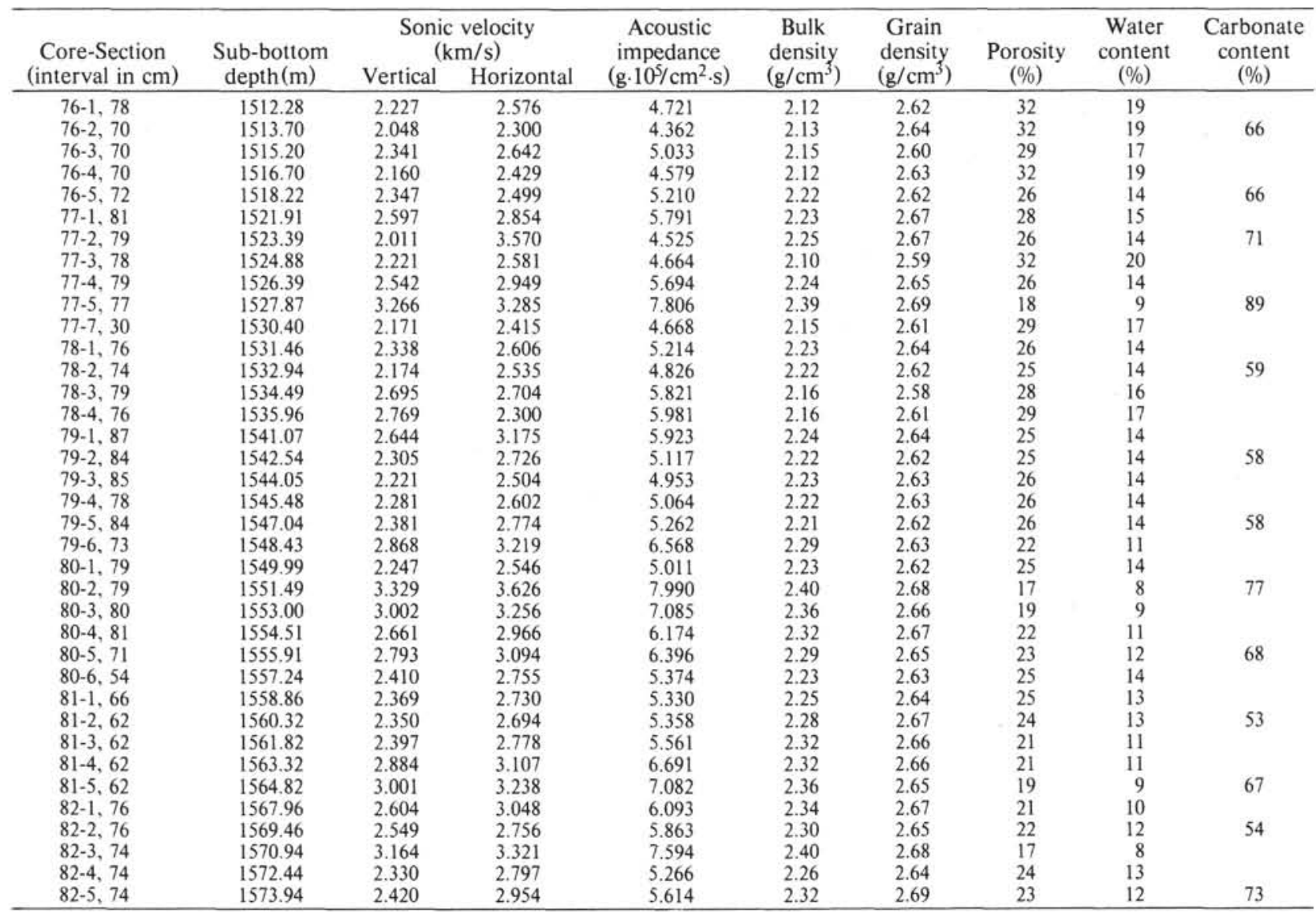


Table 3. Summary of physical properties data for samples from Hole 603C.

\begin{tabular}{|c|c|c|c|c|c|c|c|c|c|}
\hline $\begin{array}{l}\text { Core-Section } \\
\text { (interval in } \mathrm{cm} \text { ) }\end{array}$ & $\begin{array}{l}\text { Sub-bottom } \\
\text { depth }(\mathrm{m})\end{array}$ & $\begin{array}{c}\text { Vertical } \\
\text { sonic velocity } \\
(\mathrm{km} / \mathrm{s})\end{array}$ & $\begin{array}{c}\text { Acoustic } \\
\text { impedance } \\
\left(\mathrm{g} \cdot 10^{5} / \mathrm{cm}^{2} \cdot \mathrm{s}\right)\end{array}$ & $\begin{array}{l}\text { Shear } \\
\text { strength } \\
(\mathrm{kPa})\end{array}$ & $\begin{array}{c}\text { Bulk } \\
\text { density } \\
\left(\mathrm{g} / \mathrm{cm}^{3}\right)\end{array}$ & $\begin{array}{l}\text { Grain } \\
\text { density } \\
\left(\mathrm{g} / \mathrm{cm}^{3}\right)\end{array}$ & $\begin{array}{l}\text { Porosity } \\
(\%)\end{array}$ & $\begin{array}{l}\text { Water } \\
\text { content } \\
(\%)\end{array}$ & $\begin{array}{c}\text { Carbonate } \\
\text { content } \\
(\%)\end{array}$ \\
\hline $1-1,83$ & 0.83 & 1.469 & 2.321 & & 1.58 & 2.66 & 66 & 43 & 27 \\
\hline $2-1,130$ & 3.30 & 1.555 & 2.472 & 3.60 & 1.59 & 2.67 & 66 & 43 & \\
\hline $2-2,75$ & 4.25 & & & 3.60 & 1.53 & 2.72 & 70 & 47 & 25 \\
\hline $2-3,50$ & 5.50 & & & 3.60 & 1.58 & 2.82 & 69 & 45 & \\
\hline $2-4,25$ & 6.75 & 1.479 & & & & & & & \\
\hline $2-4,75$ & 7.25 & 1.531 & 2.572 & 2.25 & 1.68 & 2.83 & 64 & 39 & \\
\hline $2-4,125$ & 7.75 & 1.496 & & & & & & & \\
\hline $2-5,75$ & 8.75 & 1.483 & 2.402 & 3.42 & 1.62 & 2.80 & 67 & 42 & 25 \\
\hline $2-6,75$ & 10.25 & 1.506 & & & & & & & \\
\hline $2-6,100$ & 10.50 & 1.511 & & & & & & & \\
\hline $2-7,20$ & 11.20 & 1.545 & & & & & & & \\
\hline $2-7,75$ & 11.75 & 1.494 & & & & & & & \\
\hline $3-1,25$ & 11.85 & 1.505 & & & & & & & \\
\hline $3-1,75$ & 12.35 & 1.498 & 2.517 & 4.32 & 1.68 & 2.79 & 63 & 38 & \\
\hline $3-1,125$ & 12.85 & 1.465 & & & & & & & \\
\hline $3-2,25$ & 13.35 & 1.608 & & & & & & & \\
\hline $3-2,75$ & 13.85 & 1.499 & 2.518 & 2.88 & 1.68 & 2.52 & 57 & 35 & 18 \\
\hline $3-3,75$ & 15.35 & 1.519 & 2.537 & 2.88 & 1.67 & 2.58 & 59 & 36 & \\
\hline $3-3,125$ & 15.85 & 1.707 & & & & & & & \\
\hline $3-4,25$ & 16.35 & 1.503 & & & & & & & \\
\hline $3-4,75$ & 16.85 & 1.497 & 2.485 & 2.88 & 1.66 & 2.88 & 66 & 40 & \\
\hline $3-4,115$ & 17.25 & 1.496 & & & & & & & \\
\hline $3-5,75$ & 18.35 & & & 4.68 & 1.74 & 2.97 & 63 & 37 & 28 \\
\hline $3-6,75$ & 19.85 & 1.508 & & & & & & & \\
\hline $4-1,75$ & 21.95 & 1.489 & 2.427 & 3.42 & 1.63 & 2.73 & 65 & 41 & \\
\hline $4-1,125$ & 22.45 & 1.493 & & & & & & & \\
\hline $4-2,25$ & 22.95 & 1.480 & & & & & & & \\
\hline $4-2,75$ & 23.45 & 1.500 & & 6.84 & & & & & \\
\hline $4-3,25$ & 24.45 & 1.477 & & & & & & & \\
\hline $4-3,75$ & 24.95 & 1.503 & 2.450 & 6.30 & 1.63 & 2.74 & 65 & 41 & 26 \\
\hline $4-4,75$ & 26.45 & 1.525 & & 8.82 & & & & & \\
\hline $4-5,75$ & 27.95 & 1.492 & 2.447 & 7.56 & 1.64 & 2.74 & 64 & 40 & 31 \\
\hline $4-6,75$ & 29.45 & 1.508 & 2.549 & 8.10 & 1.69 & 2.74 & 61 & 37 & \\
\hline $5-1,50$ & 31.30 & 1.488 & & & & & & & \\
\hline $5-1,75$ & 31.55 & 1.475 & 2.286 & 6.30 & 1.55 & 2.75 & 70 & 46 & \\
\hline $5-2,50$ & 32.80 & 1.482 & & & & & & & \\
\hline $5-2,75$ & 33.05 & 1.494 & 2.241 & 8.10 & 1.50 & 2.44 & 66 & 45 & 20 \\
\hline $5-3,75$ & 34.55 & 1.496 & 2.453 & 9.90 & 1.64 & 2.90 & 67 & 42 & \\
\hline $5-4,50$ & 35.80 & 1.497 & & & & & & & \\
\hline $5-4,75$ & 36.05 & 1.499 & 2.428 & 10.44 & 1.62 & 2.68 & 64 & 41 & \\
\hline $5-5,50$ & 37.30 & 1.496 & & & & & & & \\
\hline $5-5,75$ & 37.55 & 1.501 & 2.477 & 10.44 & 1.65 & 2.78 & 64 & 40 & 28 \\
\hline $5-6,50$ & 38.80 & 1.501 & & & & & & & \\
\hline $6-1,75$ & 41.15 & 1.489 & 2.457 & 9.00 & 1.65 & 2.74 & 64 & 40 & \\
\hline $6-2,75$ & 42.65 & 1.436 & 2.341 & 8.28 & 1.63 & 2.62 & 62 & 39 & 35 \\
\hline $6-3,75$ & 44.15 & & & 9.18 & 1.65 & 2.79 & 65 & 40 & \\
\hline $6-4,75$ & 45.65 & 1.441 & 2.392 & 9.36 & 1.66 & 2.77 & 64 & 39 & \\
\hline $6-5,75$ & 47.15 & 1.470 & 2.499 & 9.90 & 1.70 & 2.84 & 63 & 38 & 16 \\
\hline $7-1,75$ & 50.65 & & & 7.20 & 1.66 & 2.73 & 63 & 39 & \\
\hline $7-2,75$ & 52.15 & & & 10.80 & 1.65 & 2.62 & 61 & 38 & 11 \\
\hline $7-3,75$ & 53.65 & & & 12.60 & 1.68 & 2.71 & 61 & 37 & \\
\hline $7-4,75$ & 55.15 & & & 10.80 & 1.70 & 2.73 & 61 & 37 & \\
\hline $7-5,75$ & 56.65 & & & 10.80 & 1.70 & 2.77 & 61 & 37 & 30 \\
\hline $8-1,75$ & 60.15 & 1.515 & 2.560 & 12.60 & 1.69 & 2.76 & 62 & 38 & \\
\hline $8-2,75$ & 61.65 & 1.500 & 2.565 & 14.58 & 1.71 & 2.89 & 63 & 38 & 15 \\
\hline $8-3,75$ & 63.15 & 1.689 & 2.905 & 14.94 & 1.72 & 2.85 & 62 & 37 & \\
\hline $8-4,75$ & 64.65 & 1.504 & 2.542 & 16.92 & 1.69 & 2.61 & 58 & 35 & \\
\hline $8-5,75$ & 66.15 & 1.512 & 2.540 & 17.10 & 1.68 & 2.73 & 62 & 38 & 3 \\
\hline $9-1,75$ & 68.55 & & & & 1.68 & 2.75 & 62 & 38 & \\
\hline $9-2,75$ & 70.05 & & & 8.46 & 1.69 & 2.85 & 63 & 38 & 12 \\
\hline $9-3,75$ & 71.55 & & & 10.62 & 1.70 & 2.79 & 62 & 37 & \\
\hline $9-4,75$ & 73.05 & & & 7.20 & 1.87 & 2.74 & 51 & 28 & \\
\hline $9-5,74$ & 74.54 & 1.532 & 2.558 & & 1.67 & 2.76 & 63 & 39 & 12 \\
\hline $10-1,80$ & 78.10 & 1.493 & 2.568 & & 1.72 & 2.94 & 63 & 38 & \\
\hline $10-2,80$ & 79.60 & 1.535 & 2.656 & & 1.73 & 2.88 & 62 & 37 & 11 \\
\hline $10-3,80$ & 81.10 & 1.508 & 2.564 & 15.84 & 1.70 & 2.77 & 62 & 37 & \\
\hline $10-4,80$ & 82.60 & 1.512 & 2.631 & 16.02 & 1.74 & 2.83 & 61 & 36 & \\
\hline $10-5,80$ & 84.10 & 1.488 & 2.515 & 15.12 & 1.69 & 2.73 & 61 & 37 & 8 \\
\hline $11-1,80$ & 85.60 & & & 12.96 & 1.71 & 2.86 & 63 & 38 & \\
\hline $11-2,80$ & 87.10 & & & 10.26 & 1.68 & 2.72 & 61 & 37 & 14 \\
\hline $11-3,80$ & 88.60 & 1.498 & 2.487 & 10.44 & 1.66 & 2.71 & 62 & 39 & \\
\hline $11-4,80$ & 90.10 & 1.515 & 2.545 & 14.04 & 1.68 & 2.71 & 61 & 37 & \\
\hline $13-1,80$ & 98.00 & & & 7.92 & 1.61 & 2.47 & 59 & 38 & \\
\hline $13-2,80$ & 99.50 & 1.451 & 2.539 & 10.26 & 1.75 & 2.92 & 62 & 36 & 12 \\
\hline $14-1,80$ & 107.60 & 1.467 & 2.421 & & 1.65 & 2.59 & 60 & 37 & \\
\hline $14-2,75$ & 109.05 & 1.513 & 2.466 & & 1.63 & 2.50 & 59 & 37 & 2 \\
\hline $14-3,75$ & 110.55 & & & & 1.65 & 2.57 & 60 & 37 & \\
\hline
\end{tabular}


Table 3 (continued).

\begin{tabular}{|c|c|c|c|c|c|c|c|c|c|}
\hline $\begin{array}{c}\text { Core-Section } \\
\text { (interval in } \mathrm{cm} \text { ) }\end{array}$ & $\begin{array}{c}\text { Sub-bottom } \\
\text { depth }(\mathrm{m})\end{array}$ & $\begin{array}{c}\text { Vertical } \\
\text { sonic velocity } \\
(\mathrm{km} / \mathrm{s})\end{array}$ & $\begin{array}{c}\text { Acoustic } \\
\text { impedance } \\
\left(\mathrm{g} \cdot 10^{5} / \mathrm{cm}^{2} \cdot \mathrm{s}\right)\end{array}$ & $\begin{array}{c}\text { Shear } \\
\text { strength } \\
(\mathrm{kPa})\end{array}$ & $\begin{array}{c}\text { Bulk } \\
\text { density } \\
\left(\mathrm{g} / \mathrm{cm}^{3}\right)\end{array}$ & $\begin{array}{l}\text { Grain } \\
\text { density } \\
\left(\mathrm{g} / \mathrm{cm}^{3}\right)\end{array}$ & $\begin{array}{c}\text { Porosity } \\
(\%)\end{array}$ & $\begin{array}{c}\text { Water } \\
\text { content } \\
(\%)\end{array}$ & $\begin{array}{c}\text { Carbonate } \\
\text { content } \\
(\%)\end{array}$ \\
\hline $14-4,75$ & 112.05 & 1.433 & 2.336 & & 1.63 & 2.51 & 59 & 37 & \\
\hline $\begin{array}{l}14-5,75 \\
14-6,75\end{array}$ & & 1.504 & 2.572 & $\begin{array}{r}9.54 \\
0.36\end{array}$ & 1.71 & 2.73 & 60 & 36 & 11 \\
\hline $\begin{array}{l}14-6,75 \\
15-1,75\end{array}$ & $\begin{array}{l}115.05 \\
117.15\end{array}$ & & & $\begin{array}{r}9.36 \\
17.10\end{array}$ & & & & 34 & \\
\hline $\begin{array}{l}15-1,75 \\
15-2,75\end{array}$ & $\begin{array}{l}117.15 \\
118.65\end{array}$ & $\begin{array}{l}1.556 \\
1.483\end{array}$ & $\begin{array}{l}2.739 \\
2.536\end{array}$ & 15.30 & 1.76 & 2.79 & $\begin{array}{l}58 \\
57\end{array}$ & $\begin{array}{l}34 \\
34\end{array}$ & \\
\hline $\begin{array}{l}15-2,75 \\
15-3,75\end{array}$ & $\begin{array}{l}118.63 \\
120.15\end{array}$ & & & 9.90 & & 2.64 & 57 & & 9 \\
\hline $\begin{array}{l}15-3,75 \\
15-4,75\end{array}$ & 121.65 & 1.544 & 2.656 & 12.06 & 1.72 & 2.72 & 59 & 35 & \\
\hline $15-5,75$ & 123.15 & 1.608 & 2.814 & 18.00 & 1.75 & 2.66 & 56 & 33 & 3 \\
\hline $16-1,75$ & 126.75 & 1.000 & 2.017 & 17.10 & 1.68 & 2.60 & 58 & 35 & \\
\hline $16-2,75$ & 128.25 & 1.497 & 2.560 & 48.60 & 1.71 & 2.65 & 58 & 35 & 2 \\
\hline $16-3,75$ & 129.75 & & & 48.60 & & & & & \\
\hline $16-4,75$ & 131.25 & 1.388 & 2.429 & 45.00 & 1.75 & 2.67 & 56 & 33 & \\
\hline $16-5,75$ & 132.75 & 1.517 & 2.700 & 48.60 & 1.78 & 2.82 & 58 & 33 & 18 \\
\hline $16-6,75$ & 134.25 & 1.437 & 2.515 & 40.00 & 1.75 & 2.72 & 57 & 33 & 8 \\
\hline $17-1,75$ & 136.35 & 1.521 & 2.586 & 32.40 & 1.70 & 2.60 & 57 & 34 & \\
\hline $17-2,75$ & 137.85 & 1.525 & 2.623 & 31.50 & 1.72 & 2.72 & 59 & 35 & 19 \\
\hline $17-3,75$ & 139.35 & 1.524 & 2.667 & 36.00 & 1.75 & 2.76 & 58 & 34 & \\
\hline $17-4,75$ & 140.85 & 1.471 & 2.515 & 43.20 & 1.71 & 2.68 & 58 & 35 & \\
\hline $17-5,75$ & 142.35 & 1.544 & 2.656 & 37.80 & 1.72 & 2.71 & 59 & 35 & \\
\hline $18-1,75$ & 145.95 & 1.508 & 2.594 & 43.20 & 1.72 & 2.69 & 58 & 35 & \\
\hline $18-2,75$ & 147.45 & 1.421 & 2.501 & 44.10 & 1.76 & 2.82 & 59 & 35 & 14 \\
\hline $18-3,75$ & 148.95 & 1.544 & 2.656 & 48.60 & 1.72 & 2.72 & 59 & 35 & \\
\hline $18-4,75$ & 150.45 & 1.495 & 2.571 & 48.60 & 1.72 & 2.68 & 58 & 35 & \\
\hline $18-5,75$ & 151.95 & 1.552 & 2.623 & 50.40 & 1.69 & 2.49 & 54 & 33 & 2 \\
\hline $19-1,75$ & 155.55 & 1.481 & 2.577 & 50.40 & 1.74 & 2.70 & 57 & 34 & \\
\hline $19-2,75$ & 157.05 & 1.503 & 2.585 & 49.50 & 1.72 & 2.61 & 56 & 33 & 5 \\
\hline $19-3,75$ & 158.55 & 1.499 & 2.563 & 52.20 & 1.71 & 2.61 & 57 & 34 & \\
\hline $19-4,75$ & 160.05 & 1.518 & 2.657 & 52.20 & 1.75 & 2.65 & 55 & 32 & \\
\hline $19-5,75$ & 161.55 & 1.545 & 2.750 & 50.40 & 1.78 & 2.69 & 55 & 32 & 12 \\
\hline $20-1,80$ & 165.20 & 1.488 & 2.634 & & 1.77 & 2.69 & 57 & 33 & \\
\hline $20-2,80$ & 166.70 & 1.514 & 2.755 & 50.40 & 1.82 & 2.97 & 59 & 33 & 13 \\
\hline $20-3,80$ & 168.20 & 1.523 & 2.696 & 52.20 & 1.77 & 2.73 & 56 & 32 & \\
\hline $20-4,80$ & 169.70 & 1.503 & 2.720 & 32.40 & 1.81 & 2.82 & 56 & 32 & \\
\hline $20-5,80$ & 171.20 & 1.500 & 2.685 & 30.60 & 1.79 & 2.86 & 59 & 34 & 15 \\
\hline $20-6,80$ & 172.70 & 1.544 & 2.717 & 61.20 & 1.76 & 2.70 & 56 & 33 & \\
\hline $21-1,80$ & 174.80 & 1.526 & 2.640 & 40.50 & 1.73 & 2.60 & 55 & 33 & \\
\hline $21-2,80$ & 176.30 & 1.503 & 2.510 & 36.00 & 1.67 & 2.46 & 55 & 34 & 16 \\
\hline $21-3,80$ & 177.80 & 1.530 & 2.555 & 37.80 & 1.67 & 2.73 & 57 & 33 & \\
\hline $21-4,80$ & 179.30 & 1.505 & 2.634 & 41.40 & 1.75 & 2.69 & 56 & 33 & \\
\hline $21-5,80$ & 180.80 & 1.538 & 2.722 & 39.60 & 1.77 & 2.78 & 58 & 33 & 9 \\
\hline $21-6,80$ & 182.30 & 1.732 & & & & & & & \\
\hline $21-7,22$ & 183.22 & 1.517 & & & & & & & \\
\hline $22-1,80$ & 184.40 & & & 18.00 & 1.70 & 2.61 & $\begin{array}{l}58 \\
57\end{array}$ & $\begin{array}{l}35 \\
33\end{array}$ & \\
\hline $22-2,80$ & 185.90 & & & 32.40 & 1.77 & 2.76 & 57 & & 7 \\
\hline $22-3,80$ & 187.40 & & & 39.60 & 1.75 & 2.70 & 56 & 33 & \\
\hline $22-4,80$ & 188.90 & & & 43.20 & 1.68 & 2.51 & 56 & 34 & \\
\hline $22-5,80$ & 190.40 & & & 36.00 & 1.80 & 2.70 & 54 & 31 & 7 \\
\hline $23-1,80$ & 194.00 & 1.529 & 2.875 & 64.80 & 1.88 & 3.22 & 61 & 33 & \\
\hline $23-2,80$ & 195.50 & 1.522 & 2.755 & 70.20 & 1.81 & 2.75 & 54 & 31 & 1 \\
\hline $23-3,80$ & 197.00 & 1.501 & 2.717 & 61.20 & 1.81 & 2.73 & 54 & 30 & \\
\hline $23-4,80$ & 198.50 & 1.518 & 2.732 & 63.00 & 1.80 & 2.72 & 54 & 31 & \\
\hline $23-5,80$ & 200.00 & 1.520 & & & & & & & \\
\hline $23-6,80$ & 201.50 & 1.510 & & & & & & & \\
\hline $24-1,80$ & 203.60 & 1.521 & 2.783 & 66.60 & 1.83 & 2.72 & 53 & 29 & \\
\hline $24-2,80$ & 205.10 & 1.536 & 2.826 & 48.60 & 1.84 & 2.85 & 56 & 31 & 19 \\
\hline $24-3,80$ & 206.60 & 1.547 & 2.723 & 63.00 & 1.76 & 2.58 & 53 & 31 & \\
\hline $24-4,80$ & 208.10 & 1.474 & 2.668 & 64.80 & 1.81 & 2.72 & 54 & 30 & \\
\hline $24-5,80$ & 209.60 & 1.538 & 2.784 & 66.60 & 1.81 & 2.67 & 52 & 30 & 4 \\
\hline $25-1,80$ & 213.20 & 1.531 & 2.740 & 43.20 & 1.79 & 2.54 & 50 & 29 & \\
\hline $25-2,80$ & 214.70 & 1.499 & 2.743 & 59.40 & 1.83 & 2.78 & 54 & 30 & 7 \\
\hline $25-3,80$ & 216.20 & 1.520 & 2.706 & 68.40 & 1.78 & 2.59 & 52 & 30 & \\
\hline $25-4,80$ & 217.70 & 1.517 & 2.746 & 50.40 & 1.81 & 2.71 & 53 & 30 & \\
\hline $25-5,80$ & 219.20 & 1.095 & 1.851 & 52.20 & 1.69 & 2.42 & 52 & 32 & 4 \\
\hline $25-6,80$ & 220.70 & 1.517 & & & & & & & \\
\hline $25-6,80$ & 220.70 & 1.583 & & & & & & & \\
\hline $26-1,80$ & 222.80 & 1.583 & 2.818 & 54.90 & 1.78 & 2.69 & 55 & 31 & \\
\hline $26-2,80$ & 224.30 & 1.488 & 2.678 & 61.20 & 1.80 & 2.68 & 53 & 30 & 8 \\
\hline $26-3,80$ & 225.80 & & & 64.80 & & & & & \\
\hline $26-4,75$ & 227.25 & & & 55.80 & 1.77 & 2.61 & 53 & 31 & \\
\hline $26-5,75$ & 228.75 & & & 57.60 & 1.76 & 2.59 & 53 & 31 & 14 \\
\hline $27-1,75$ & 232.35 & 1.440 & 2.563 & 40.50 & 1.78 & 2.71 & 55 & 32 & \\
\hline $27-2,75$ & 233.85 & 1.477 & 2.629 & 63.90 & 1.78 & 2.61 & 52 & 30 & 9 \\
\hline $27-3,75$ & 235.35 & 1.441 & 2.637 & 73.80 & 1.83 & 2.80 & 55 & 31 & \\
\hline $27-4,75$ & 236.85 & 1.443 & 2.583 & 81.00 & 1.79 & 2.59 & 51 & 29 & \\
\hline $27-5,75$ & 238.35 & 1.527 & 2.779 & 81.00 & 1.82 & 2.62 & 52 & 30 & 4 \\
\hline $28-1,75$ & 241.95 & 1.497 & 2.635 & 54.00 & 1.76 & 2.72 & 56 & 33 & \\
\hline $28-2,75$ & 243.45 & 1.502 & 2.674 & 54.00 & 1.78 & 2.65 & 53 & 31 & 7 \\
\hline
\end{tabular}


Table 3 (continued).

\begin{tabular}{|c|c|c|c|c|c|c|c|c|c|}
\hline $\begin{array}{c}\text { Core-Section } \\
\text { (interval in } \mathrm{cm} \text { ) }\end{array}$ & $\begin{array}{c}\text { Sub-bottom } \\
\text { depth }(\mathrm{m})\end{array}$ & $\begin{array}{c}\text { Vertical } \\
\text { sonic velocity } \\
(\mathrm{km} / \mathrm{s})\end{array}$ & $\begin{array}{c}\text { Acoustic } \\
\text { impedance } \\
\left(\mathrm{g} \cdot 10^{5} / \mathrm{cm}^{2} \cdot \mathrm{s}\right)\end{array}$ & $\begin{array}{l}\text { Shear } \\
\text { strength } \\
(\mathrm{kPa})\end{array}$ & $\begin{array}{c}\text { Bulk } \\
\text { density } \\
\left(\mathrm{g} / \mathrm{cm}^{3}\right)\end{array}$ & $\begin{array}{c}\text { Grain } \\
\text { density } \\
\left(\mathrm{g} / \mathrm{cm}^{3}\right)\end{array}$ & $\begin{array}{c}\text { Porosity } \\
(\%)\end{array}$ & $\begin{array}{c}\text { Water } \\
\text { content } \\
(\%)\end{array}$ & $\begin{array}{c}\text { Carbonate } \\
\text { content } \\
(\%)\end{array}$ \\
\hline $28-3,75$ & 244.95 & 1.501 & 2.717 & 57.60 & 1.81 & 2.75 & 54 & 31 & \\
\hline $28-4,75$ & 246.45 & 1.495 & 2.661 & 70.20 & 1.78 & 2.74 & 56 & 32 & \\
\hline $28-5,75$ & 247.95 & 1.509 & 2.716 & 77.40 & 1.80 & 2.65 & 52 & 30 & 8 \\
\hline $30-2,75$ & 262.65 & 1.386 & 2.467 & 72.00 & 1.78 & 2.51 & 49 & 28 & 2 \\
\hline $31-3,75$ & 273.75 & 1.128 & 2.030 & 68.40 & 1.80 & 2.64 & 52 & 30 & \\
\hline $31-4,75$ & 275.25 & & 2.070 & 68.40 & 1.76 & 2.53 & 51 & 30 & \\
\hline $31-5,75$ & 276.75 & & & 68.40 & 1.83 & 2.70 & 52 & 29 & 2 \\
\hline $33-2,84$ & 291.54 & 1.571 & 2.844 & 63.00 & 1.81 & 2.59 & 50 & 28 & 2 \\
\hline $33-3,71$ & 292.91 & 1.543 & 2.731 & & 1.77 & 2.62 & 53 & 31 & \\
\hline $33-4,78$ & 294.48 & 1.537 & 2.736 & 76.50 & 1.78 & 2.60 & 52 & 30 & \\
\hline $33-5,70$ & 295.90 & 1.663 & 3.010 & 79.20 & 1.81 & 2.55 & 49 & 28 & 5 \\
\hline $33-6,70$ & 297.40 & 1.545 & 2.627 & & 1.70 & 2.50 & 54 & 33 & \\
\hline $34-1,124$ & 300.04 & 1.552 & & & & & & & 14 \\
\hline $35-1,79$ & 309.19 & 1.561 & 2.732 & 45.00 & 1.75 & 2.57 & 53 & 31 & \\
\hline $35-2,72$ & 310.62 & 1.460 & 2.628 & 70.20 & 1.80 & 2.73 & 55 & 31 & 9 \\
\hline $35-3,80$ & 312.20 & 1.576 & 2.774 & 72.00 & 1.76 & 2.52 & 51 & 30 & \\
\hline $35-4,73$ & 313.63 & 1.614 & 2.905 & 77.40 & 1.80 & 2.63 & 52 & 30 & \\
\hline $35-5,73$ & 315.13 & 1.517 & 2.700 & 45.00 & 1.78 & 2.65 & 53 & 31 & 3 \\
\hline $35-6,76$ & 316.66 & 1.565 & & & & & & & \\
\hline $36-1,79$ & 318.79 & 1.503 & 2.735 & & 1.82 & 2.64 & 51 & 29 & \\
\hline $36-2,72$ & 320.22 & 1.568 & 2.822 & 77.40 & 1.80 & 2.68 & 53 & 30 & 7 \\
\hline $36-3,79$ & 321.79 & 1.574 & 2.723 & & 1.73 & 2.50 & 52 & 31 & \\
\hline $36-4,79$ & 323.29 & 1.588 & 2.843 & & 1.79 & 2.62 & 52 & 30 & \\
\hline $36-5,79$ & 324.79 & 1.528 & 2.720 & 73.80 & 1.78 & 2.48 & 48 & 28 & 4 \\
\hline $37-1,80$ & 328.40 & 1.618 & 2.945 & & 1.82 & 2.60 & 49 & 27 & \\
\hline $37-2,80$ & 329.90 & 1.558 & 2.820 & 79.20 & 1.81 & 2.57 & 49 & 28 & 6 \\
\hline $37-3,79$ & 331.39 & 1.546 & 2.891 & & 1.87 & 2.73 & 50 & 27 & \\
\hline $37-4,79$ & 332.89 & 1.587 & 2.872 & & 1.81 & 2.70 & 53 & 28 & \\
\hline $37-5,79$ & 334.39 & 1.576 & 2.916 & & 1.85 & 2.74 & 51 & 28 & 2 \\
\hline $37-6,80$ & 335.90 & 1.592 & & & & & & & \\
\hline $38-1,82$ & 338.02 & & & & 1.80 & 2.70 & 54 & 31 & \\
\hline $38-2,82$ & 339.52 & 1.563 & 2.845 & & 1.82 & 2.59 & 49 & 28 & 4 \\
\hline $38-3,92$ & 341.12 & 1.901 & 3.403 & & 1.79 & 2.55 & 50 & 29 & \\
\hline $38-4,82$ & 342.52 & 1.570 & 3.046 & & 1.94 & 2.73 & 46 & 25 & 20 \\
\hline $38-6,80$ & 345.50 & & & & 1.81 & 2.61 & 50 & 28 & \\
\hline $39-1,68$ & 347.48 & 1.518 & 2.717 & & 1.79 & 2.57 & 50 & 29 & \\
\hline $39-2,73$ & 349.03 & 1.516 & 2.714 & & 1.79 & 2.51 & 48 & 28 & 11 \\
\hline $39-3,75$ & 350.55 & 1.540 & 2.849 & & 1.85 & 2.64 & 49 & 27 & \\
\hline $39-4,75$ & 352.05 & 1.491 & 2.729 & & 1.83 & 2.61 & 49 & 28 & \\
\hline $39-5,75$ & 353.55 & 1.469 & 2.806 & & 1.91 & 2.68 & 47 & 25 & 2 \\
\hline $40-1,75$ & 357.15 & 1.591 & & & & & & & \\
\hline $40-2,75$ & 358.65 & 1.551 & 2.745 & & 1.77 & 2.40 & 46 & 26 & 3 \\
\hline $40-3,75$ & 360.15 & 1.591 & & & & & & & \\
\hline $40-4,75$ & 361.65 & 1.575 & & & & & & & \\
\hline $40-5,75$ & 363.15 & 1.592 & 2.945 & & 1.85 & 2.67 & 50 & 28 & 14 \\
\hline
\end{tabular}


Table 4. Summary of physical properties data for samples from Hole 604 .

\begin{tabular}{|c|c|c|c|c|c|c|c|c|c|c|}
\hline $\begin{array}{l}\text { Core-Section } \\
\text { (interval in cm) }\end{array}$ & $\begin{array}{l}\text { Sub-bottom } \\
\text { depth }(m)\end{array}$ & $\begin{array}{c}\text { Vertical } \\
\text { sonic velocity } \\
(\mathrm{km} / \mathrm{s})\end{array}$ & $\begin{array}{c}\text { Acoustic } \\
\text { impedance } \\
\left(\mathrm{g} \cdot 10^{5} / \mathrm{cm}^{2} \cdot \mathrm{s}\right)\end{array}$ & $\begin{array}{l}\text { Shear } \\
\text { strength } \\
(\mathrm{kPa})\end{array}$ & $\begin{array}{l}\text { Unconfined } \\
\text { compression } \\
\text { strength } \\
(\mathrm{kPa})\end{array}$ & $\begin{array}{c}\text { Bulk } \\
\text { density } \\
\left(\mathrm{g} / \mathrm{cm}^{3}\right)\end{array}$ & $\begin{array}{l}\text { Grain } \\
\text { density } \\
\left(\mathrm{g} / \mathrm{cm}^{3}\right)\end{array}$ & $\begin{array}{c}\text { Porosity } \\
(\%)\end{array}$ & $\begin{array}{c}\text { Water } \\
\text { content } \\
(\%)\end{array}$ & $\begin{array}{c}\text { Carbonate } \\
\text { content } \\
(\%) \\
\end{array}$ \\
\hline $1-2,75$ & 2.25 & & & 8.64 & & 1.85 & 2.75 & 53 & 29 & 14 \\
\hline $1-3,75$ & 3.75 & & & 5.94 & & 2.06 & 2.82 & 43 & 21 & 10 \\
\hline $2-1,75$ & 7.25 & 1.763 & 3.632 & 3.96 & & 2.06 & 2.93 & 46 & 23 & 10 \\
\hline $3-1,75$ & 16.85 & 1.652 & 3.271 & & 27.44 & 1.98 & 2.81 & 47 & 24 & 8 \\
\hline $5-1,75$ & 36.05 & 1.637 & 3.143 & 17.10 & 17.64 & 1.92 & 2.72 & 47 & 25 & \\
\hline $5-2,75$ & 37.55 & 1.663 & 3.259 & 12.24 & 6.86 & 1.96 & 2.78 & 47 & 24 & 10 \\
\hline $6-1,75$ & 45.65 & 1.752 & 3.469 & 16.20 & & 1.98 & 2.74 & 45 & 23 & \\
\hline $6-2,75$ & 47.15 & 1.532 & 2.666 & 12.24 & & 1.74 & 2.49 & 52 & 30 & 5 \\
\hline $6-3,75$ & 48.65 & 1.627 & 2.815 & 11.70 & & 1.73 & 2.68 & 57 & 34 & \\
\hline $6-4,75$ & 50.15 & 1.577 & 3.249 & 14.40 & & 2.06 & 2.78 & 41 & 21 & \\
\hline $6-5,25$ & 51.15 & 1.524 & 2.713 & 14.40 & & 1.78 & 2.68 & 55 & 31 & 8 \\
\hline $8-1,79$ & 64.89 & 1.600 & 3.200 & 12.96 & 17.64 & 2.00 & 2.73 & 43 & 22 & \\
\hline $8-2,68$ & 66.28 & 1.570 & 3.093 & 10.62 & 15.68 & 1.97 & 2.70 & 44 & 23 & 5 \\
\hline $9-1,75$ & 74.45 & & & & & 1.85 & 2.79 & 53 & 30 & \\
\hline $9-2,75$ & 75.95 & 1.543 & 2.870 & 9.18 & 14.70 & 1.86 & 2.68 & 49 & 27 & \\
\hline $10-1,82$ & 84.12 & 1.550 & 2.511 & 5.22 & 12.74 & 1.62 & 2.59 & 62 & 40 & \\
\hline $10-2,82$ & 85.62 & 1.646 & 2.765 & 2.34 & 3.92 & 1.68 & 2.62 & 59 & 36 & 9 \\
\hline $10-3,82$ & 87.12 & 1.247 & 2.207 & 5.76 & 12.74 & 1.77 & 3.10 & 64 & 37 & \\
\hline $10-4,82$ & 88.62 & 1.547 & 2.444 & 4.14 & 12.25 & 1.58 & 2.52 & 63 & 40 & \\
\hline $10-5,9$ & 89.39 & 1.571 & & & 17.64 & & & & & \\
\hline $10, \mathrm{CC}$ & 89.82 & & & & & 1.90 & 2.57 & 43 & 23 & \\
\hline $11-1,72$ & 93.62 & & & 7.02 & 9.80 & 1.75 & 2.52 & 52 & 30 & \\
\hline $11-2,72$ & 95.12 & & & 6.66 & 17.64 & 1.75 & 2.44 & 49 & 29 & 4 \\
\hline $11-3,72$ & 96.62 & & & 7.02 & 16.66 & 1.88 & 2.72 & 49 & 27 & \\
\hline $11-4,72$ & 98.12 & 1.669 & 3.038 & 6.66 & 18.62 & 1.82 & 2.54 & 47 & 27 & \\
\hline $11-5.74$ & 99.64 & 1.552 & 3.120 & & 28.42 & 2.01 & 2.69 & 41 & 21 & 8 \\
\hline $12-1,82$ & 103.32 & 1.478 & 2.734 & 10.44 & 13.72 & 1.85 & 2.69 & 50 & 28 & 9 \\
\hline $13-1,73$ & 112.83 & 1.610 & 3.252 & 11.34 & 18.13 & 2.02 & 2.72 & 42 & 21 & \\
\hline $13-2,73$ & 114.33 & 1.558 & 2.695 & & 21.56 & 1.73 & 2.51 & 52 & 31 & 7 \\
\hline $13-3,73$ & 115.83 & & & 5.40 & 17.64 & 1.68 & 2.66 & 60 & 37 & \\
\hline $13-4,73$ & 117.33 & 1.547 & 2.382 & 7.38 & 17.15 & 1.54 & 2.38 & 62 & 41 & \\
\hline $14-1,73$ & 122.43 & 1.525 & 3.004 & 6.66 & 26.46 & 1.97 & 2.73 & 45 & 23 & \\
\hline $14-2,73$ & 123.93 & & & 10.44 & 23.52 & 1.69 & 2.58 & 57 & 35 & 9 \\
\hline $14-3,73$ & 125.43 & 1.542 & 2.668 & 7.92 & 18.62 & 1.73 & 2.45 & 51 & 30 & 8 \\
\hline $14-4,73$ & 126.93 & 1.587 & 2.682 & 5.40 & 21.56 & 1.69 & 2.56 & 57 & 34 & \\
\hline $14-5,79$ & 128.49 & 1.630 & 2.771 & 7.02 & 26.46 & 1.70 & 2.63 & 58 & 35 & 13 \\
\hline $15-1,73$ & 132.03 & & & 7.74 & 14.70 & 1.82 & 2.65 & 51 & 29 & \\
\hline $15-2,73$ & 133.53 & 1.695 & 3.170 & 8.28 & 22.54 & 1.87 & 2.59 & 46 & 25 & 19 \\
\hline $15-3,15$ & 134.45 & 1.510 & 2.673 & 8.46 & 22.54 & 1.77 & 2.63 & 53 & 31 & 12 \\
\hline $16-1,73$ & 141.63 & 1.525 & 2.806 & 9.54 & 11.27 & 1.84 & 2.64 & 50 & 28 & \\
\hline $16-2,73$ & 143.13 & 1.556 & 2.941 & 15.84 & 20.58 & 1.89 & 2.72 & 49 & 27 & 7 \\
\hline $16-3,73$ & 144.63 & 1.534 & 2.669 & & 24.50 & 1.74 & 2.56 & 54 & 32 & \\
\hline $16-4,73$ & 146.13 & 1.572 & 2.782 & 9.72 & 25.48 & 1.77 & 2.59 & 53 & 31 & 6 \\
\hline $17-1,73$ & 151.23 & 1.556 & 2.723 & 15.12 & 29.40 & 1.75 & 2.53 & 52 & 30 & \\
\hline $17-2,73$ & 152.73 & 1.565 & 2.707 & 5.76 & 17.64 & 1.73 & 2.51 & 52 & 31 & 19 \\
\hline $17-3,73$ & 154.23 & 1.694 & 2.761 & 8.10 & 21.56 & 1.63 & 2.48 & 58 & 37 & \\
\hline $17-4,73$ & 155.73 & 1.518 & 2.672 & 14.94 & 19.60 & 1.76 & 2.57 & 52 & 30 & 8 \\
\hline $18-1,73$ & 160.83 & 1.567 & 2.789 & 18.00 & 25.48 & 1.78 & 2.69 & 55 & 31 & \\
\hline $18-2,73$ & 162.33 & 1.516 & 2.835 & 18.00 & 33.32 & 1.87 & 2.61 & 47 & 26 & 11 \\
\hline $18-3,73$ & 163.83 & & & & 44.10 & 1.84 & 2.66 & 50 & 28 & \\
\hline $18-4,73$ & 165.33 & & & 12.24 & 26.46 & 1.70 & 2.45 & 52 & 32 & 4 \\
\hline $19-1,75$ & 170.45 & 1.418 & 2.396 & 12.60 & 19.60 & 1.69 & 2.39 & 51 & 31 & \\
\hline $19-2,75$ & 171.95 & 1.543 & 2.793 & 5.58 & 18.72 & 1.81 & 2.73 & 54 & 31 & 2 \\
\hline $19-3,75$ & 173.45 & 1.385 & & & 39.20 & & & & & \\
\hline $19-4,75$ & 174.95 & 1.500 & 2.520 & & 39.20 & 1.68 & 2.41 & 53 & 32 & 13 \\
\hline $20-1,75$ & 180.05 & 1.493 & 2.583 & & 39.20 & 1.73 & 2.53 & 53 & 31 & \\
\hline $20-2,75$ & 181.55 & 1.410 & 2.369 & & 35.28 & 1.68 & 2.44 & 54 & 33 & 8 \\
\hline $21-1,75$ & 189.65 & 1.421 & 2.345 & & 41.65 & 1.65 & 2.29 & 51 & 32 & \\
\hline $21-2,75$ & 191.15 & 1.422 & 2.574 & & 44.10 & 1.81 & 2.66 & 52 & 30 & 7 \\
\hline $21-3,75$ & 192.65 & 1.446 & 2.502 & & 44.10 & 1.73 & 2.38 & 47 & 28 & \\
\hline $21-4,75$ & 194.15 & 1.358 & 2.512 & & 39.20 & 1.85 & 2.54 & 45 & 25 & \\
\hline $22-1,75$ & 199.25 & 1.370 & 2.329 & & 37.24 & 1.70 & 2.44 & 52 & 31 & \\
\hline $22-2,75$ & 200.75 & 1.510 & 2.416 & & 38.22 & 1.60 & 2.11 & 47 & 30 & 9 \\
\hline $22-3,75$ & 202.25 & 1.451 & 2.394 & & 41.16 & 1.65 & 2.55 & 50 & 28 & \\
\hline $22-4,75$ & 203.75 & 1.485 & 2.658 & & 41.16 & 1.79 & 2.52 & 49 & 28 & \\
\hline $22-5,75$ & 205.25 & 1.429 & 2.301 & & 37.24 & 1.61 & 2.31 & 54 & 34 & 9 \\
\hline $23-1,75$ & 208.85 & 2.039 & 3.507 & & 32.34 & 1.72 & 2.57 & 55 & 33 & \\
\hline $23-2,75$ & 210.35 & 1.535 & 2.502 & & 27.44 & 1.63 & 2.51 & 59 & 37 & 12 \\
\hline $23-3,75$ & 211.85 & 1.381 & 2.279 & & 42.14 & 1.65 & 2.44 & 56 & 35 & \\
\hline $25-1,75$ & 228.05 & 1.467 & 2.479 & & 44.10 & 1.69 & 2.44 & 53 & 32 & \\
\hline $25-2,75$ & 229.55 & 1.432 & 2.406 & & 44.10 & 1.68 & 2.30 & 49 & 30 & 5 \\
\hline $25-3,75$ & 231.05 & 1.552 & 2.514 & & 37.24 & 1.62 & 2.41 & 57 & 36 & \\
\hline $25-4,75$ & 232.55 & & & & 24.50 & 1.70 & 2.44 & 52 & 31 & 3 \\
\hline $26-1,75$ & 237.65 & 1.237 & 2.041 & & 24.50 & 1.65 & 2.36 & 53 & 33 & \\
\hline $26-2,75$ & 239.15 & 1.421 & 2.359 & & 24.50 & 1.66 & 2.28 & 49 & 31 & 2 \\
\hline $26-3,75$ & 240.65 & 1.460 & 2.482 & & 24.50 & 1.70 & 2.33 & 49 & 29 & \\
\hline $27-1,75$ & 247.25 & 1.518 & 2.292 & & & 1.51 & 2.05 & 52 & 35 & 59 \\
\hline $28-1,75$ & 256.85 & 1.450 & 2.581 & 9.00 & 14.70 & 1.78 & 2.58 & 52 & 30 & \\
\hline
\end{tabular}


Table 5. Summary of physical properties data for samples from Hole 605 .

\begin{tabular}{|c|c|c|c|c|c|c|c|c|c|c|}
\hline $\begin{array}{l}\text { Core-Section } \\
\text { (interval in cm) }\end{array}$ & $\begin{array}{c}\text { Sub-bottom } \\
\text { depth }(m)\end{array}$ & \multicolumn{2}{|c|}{$\begin{array}{l}\text { Sonic velocity } \\
(\mathrm{km} / \mathrm{s})\end{array}$} & $\begin{array}{c}\text { Acoustic } \\
\text { impedance } \\
\left(\mathrm{g} \cdot 10^{5} / \mathrm{cm}^{2} \cdot \mathrm{s}\right)\end{array}$ & $\begin{array}{l}\text { Unconfined } \\
\text { compression } \\
\text { strength } \\
\text { (kPa) }\end{array}$ & $\begin{array}{l}\text { Bulk } \\
\text { density } \\
\left(\mathrm{g} / \mathrm{cm}^{3}\right)\end{array}$ & $\begin{array}{l}\text { Grain } \\
\text { density } \\
\left(\mathrm{g} / \mathrm{cm}^{3}\right)\end{array}$ & $\begin{array}{l}\text { Porosity } \\
(\%)\end{array}$ & $\begin{array}{l}\text { Water } \\
\text { content } \\
(\%)\end{array}$ & $\begin{array}{c}\text { Carbonate } \\
\text { content } \\
(\%)\end{array}$ \\
\hline $3-1,75$ & 164.65 & 1.243 & & 2.474 & 24.50 & 1.99 & 2.70 & 42 & 21 & 12 \\
\hline $3-2,75$ & 166.15 & 1.584 & & 2.962 & 25.48 & 1.87 & 2.69 & 49 & 21 & \\
\hline $3-3,75$ & 167.65 & 1.675 & & 3.400 & 44.10 & 2.03 & 2.73 & 41 & 20 & 8 \\
\hline $4-1,75$ & 174.25 & 1.577 & & 3.264 & 39.20 & 2.07 & 2.74 & 39 & 19 & 10 \\
\hline $4-2,75$ & 175.75 & 1.511 & & 3.203 & 29.40 & 2.12 & 2.73 & 36 & 17 & \\
\hline $4-3,75$ & 177.25 & 1.659 & & 3.550 & 34.30 & 2.14 & 2.83 & 38 & 18 & \\
\hline $5-1,75$ & 183.85 & 1.469 & & 3.129 & 41.65 & 2.13 & 2.76 & 36 & 17 & 18 \\
\hline $5-2,75$ & 185.35 & 1.606 & & 3.469 & 44.10 & 2.16 & 2.76 & 34 & 16 & \\
\hline $6-1,75$ & 193.45 & 1.669 & & 3.438 & 44.10 & 2.06 & 2.63 & 35 & 17 & 9 \\
\hline $6-2,75$ & 194.95 & 1.652 & & 3.354 & 44.10 & 2.03 & 2.56 & 34 & 17 & \\
\hline $6-3,75$ & 196.45 & 1.678 & & 3.574 & 44.10 & 2.13 & 2.71 & 34 & 16 & 9 \\
\hline $7-1,75$ & 203.05 & 1.623 & & 2.645 & & 1.63 & 2.40 & 57 & 60 & \\
\hline $7-3,75$ & 206.05 & & & & & 1.60 & 2.35 & 58 & 63 & 47 \\
\hline $7-4,75$ & 207.55 & 1.671 & 1.735 & 2.690 & & 1.61 & 2.36 & 58 & 62 & \\
\hline $7-5,75$ & 209.05 & 1.713 & 1.713 & 2.775 & & 1.62 & 2.37 & 57 & 61 & 51 \\
\hline $7-6,75$ & 210.55 & 1.593 & 1.676 & 2.485 & & 1.56 & 2.30 & 60 & 69 & \\
\hline $9-1,75$ & 222.25 & 1.738 & 1.814 & 2.989 & & 1.72 & 2.45 & 52 & 48 & 60 \\
\hline $9-2,75$ & 223.75 & 1.740 & 1.803 & 3.062 & & 1.76 & 2.52 & 52 & 46 & \\
\hline $9.3,75$ & 225.25 & 1.758 & 1.797 & 3.059 & & 1.74 & 2.47 & 52 & 46 & 61 \\
\hline $9-4,75$ & 226.75 & 1.754 & 1.841 & 3.017 & & 1.72 & 2.50 & 54 & 50 & \\
\hline $10-1,77$ & 231.87 & 1.699 & 1.703 & 2.752 & & 1.62 & 2.38 & 58 & 61 & \\
\hline $10-2,77$ & 233.37 & 1.703 & 1.742 & 2.759 & & 1.62 & 2.38 & 57 & 60 & \\
\hline $10-3,77$ & 234.87 & 1.655 & 1.674 & 2.648 & & 1.60 & 2.38 & 59 & 65 & \\
\hline $10-4,77$ & 236.37 & 1.651 & 1.681 & 2.675 & & 1.62 & 2.41 & 58 & 62 & \\
\hline $10-5,77$ & 237.87 & 1.664 & 1.659 & 2.729 & & 1.64 & 2.44 & 58 & 60 & \\
\hline $11-1,75$ & 241.45 & 1.648 & 1.696 & 2.521 & & 1.53 & 2.29 & 62 & 75 & \\
\hline $11-2,75$ & 242.95 & 1.631 & 1.687 & 2.495 & & 1.53 & 2.30 & 62 & 76 & \\
\hline $11-3,77$ & 244.47 & 1.657 & 1.667 & 2.486 & & 1.50 & 2.25 & 63 & 82 & \\
\hline $11-4,77$ & 245.97 & 1.630 & 1.648 & 2.412 & & 1.48 & 2.23 & 64 & 86 & \\
\hline $11-5,77$ & 247.47 & 1.703 & 1.743 & 2.810 & & 1.65 & 2.39 & 56 & 56 & \\
\hline $12-1,75$ & 251.05 & 1.661 & 1.735 & 2.674 & & 1.61 & 2.39 & 59 & 64 & \\
\hline $12-2,75$ & 252.55 & 1.662 & 1.639 & 2.510 & & 1.51 & 2.27 & 63 & 79 & \\
\hline $12-3,75$ & 254.05 & 1.753 & 1.766 & 2.875 & & 1.64 & 2.36 & 55 & 55 & \\
\hline $12-4,75$ & 255.55 & 1.635 & 1.730 & 2.600 & & 1.59 & 2.10 & 49 & 49 & \\
\hline $12-4,75$ & 255.55 & 1.635 & 1.730 & 2.600 & & 1.59 & 2.10 & 49 & 49 & \\
\hline $12-5,75$ & 257.05 & 1.662 & 1.721 & 2.593 & & 1.56 & 2.36 & 61 & 71 & \\
\hline $13-1,75$ & 260.65 & 1.832 & 1.851 & 3.169 & & 1.73 & 2.44 & 52 & 47 & \\
\hline $13-2,75$ & 262.15 & 1.792 & 1.823 & 3.136 & & 1.75 & 2.48 & 51 & 45 & \\
\hline $13-3,75$ & 263.65 & 1.720 & 1.780 & 2.804 & & 1.63 & 2.61 & 63 & 70 & \\
\hline $13-4,75$ & 265.15 & 1.677 & 1.726 & 2.767 & & 1.65 & 2.40 & 56 & 56 & \\
\hline $13-5,75$ & 266.65 & 1.726 & 1.687 & 2.813 & & 1.63 & 2.38 & 57 & 59 & \\
\hline $14-1,75$ & 270.25 & 1.677 & 1.730 & 2.784 & & 1.66 & 2.42 & 56 & 55 & \\
\hline $14-2,75$ & 271.75 & 1.642 & 1.712 & 2.742 & & 1.67 & 2.46 & 56 & 56 & \\
\hline $14-3,75$ & 273.25 & 1.719 & 1.749 & 2.836 & & 1.65 & 2.43 & 57 & 58 & \\
\hline $14-4,75$ & 274.75 & 1.649 & 1.782 & 2.770 & & 1.68 & 2.45 & 55 & 54 & \\
\hline $14-5,75$ & 276.25 & 1.703 & 1.680 & 2.912 & & 1.71 & 2.47 & 54 & 51 & \\
\hline $15-1,75$ & 279.85 & 1.813 & 1.789 & 3.064 & & 1.69 & 2.45 & 54 & 52 & \\
\hline $15-2,75$ & 281.35 & 1.777 & 1.855 & 3.039 & & 1.71 & 2.46 & 54 & 50 & \\
\hline $15-3,75$ & 282.85 & 1.770 & 1.759 & 2.850 & & 1.61 & 2.38 & 58 & 63 & \\
\hline $15-4,75$ & 284.35 & 1.714 & 1.778 & 2.845 & & 1.66 & 2.41 & 56 & 56 & \\
\hline $15-5,75$ & 285.85 & 1.745 & 1.781 & 2.879 & & 1.65 & 2.42 & 56 & 57 & \\
\hline $16-1,75$ & 289.45 & 1.753 & 1.785 & 2.928 & & 1.67 & 2.42 & 55 & 54 & \\
\hline $16-2,75$ & 290.95 & 1.694 & 1.781 & 2.727 & & 1.61 & 2.38 & 58 & 63 & \\
\hline $16-3,75$ & 292.45 & 1.704 & 1.786 & 2.863 & & 1.68 & 2.40 & 54 & 52 & \\
\hline $16-4,75$ & 293.95 & 1.750 & 1.799 & 2.800 & & 1.60 & 2.47 & 62 & 70 & \\
\hline $16-5,75$ & 295.45 & 1.758 & 1.664 & 2.918 & & 1.66 & 2.42 & 56 & 55 & \\
\hline $17-1,75$ & 299.05 & 1.967 & 2.031 & 3.265 & & 1.66 & 2.42 & 56 & 55 & \\
\hline $17-2,75$ & 300.55 & 2.011 & 2.144 & 3.338 & & 1.66 & 2.42 & 56 & 56 & \\
\hline $17-3,75$ & 302.05 & 1.898 & 1.983 & 3.170 & & 1.67 & 2.40 & 55 & 53 & \\
\hline $17-4,75$ & 303.55 & 1.943 & 2.059 & 3.264 & & 1.68 & 2.43 & 55 & 53 & \\
\hline $17-5,75$ & 305.05 & 1.949 & 1.946 & 3.274 & & 1.68 & 2.41 & 54 & 52 & \\
\hline $18-1,75$ & 308.65 & 1.758 & 1.859 & 3.006 & & 1.71 & 2.44 & 53 & 49 & \\
\hline $18-2,75$ & 310.15 & 1.777 & 1.804 & 3.110 & & 1.75 & 2.48 & 52 & 46 & 55 \\
\hline $18-3,75$ & 311.65 & 1.695 & 1.766 & 2.763 & & 1.63 & 2.34 & 56 & 57 & \\
\hline $19-1,75$ & 318.25 & 1.752 & 1.849 & 3.066 & & 1.75 & 2.42 & 50 & 43 & \\
\hline $19-2,75$ & 319.75 & 1.721 & 1.779 & 2.926 & & 1.70 & 2.44 & 53 & 50 & 49 \\
\hline $19-3,75$ & 321.25 & 1.741 & 1.799 & 3.029 & & 1.74 & 2.42 & 50 & 44 & \\
\hline $19-4,75$ & 322.75 & 1.884 & 1.859 & 3.485 & & 1.85 & 2.49 & 45 & 35 & 56 \\
\hline $19-5,75$ & 324.25 & 1.815 & 1.909 & 3.321 & & 1.83 & 2.51 & 47 & 38 & \\
\hline $20-1,75$ & 327.85 & 1.721 & 1.790 & 2.840 & & 1.65 & 2.36 & 55 & 54 & \\
\hline $20-2,75$ & 329.35 & 1.787 & 1.824 & 3.074 & & 1.72 & 2.41 & 51 & 46 & \\
\hline $20-3,75$ & 330.85 & 1.723 & 1.773 & 2.912 & & 1.69 & 2.38 & 52 & 49 & 55 \\
\hline $21-1,75$ & 337.45 & 1.762 & 1.868 & 3.084 & & 1.75 & 2.39 & 48 & 41 & 49 \\
\hline $21-2,75$ & 338.95 & 1.799 & 1.854 & 3.256 & & 1.81 & 2.45 & 46 & 37 & 60 \\
\hline $21-3,75$ & 340.45 & 1.799 & 1.854 & 3.256 & & 1.81 & 2.45 & 46 & 37 & \\
\hline $21-3,75$ & 340.45 & 1.877 & 1.979 & 3.566 & & 1.90 & 2.49 & 41 & 30 & \\
\hline
\end{tabular}


Table 5 (continued).

\begin{tabular}{|c|c|c|c|c|c|c|c|c|c|c|}
\hline $\begin{array}{c}\text { Core-Section } \\
\text { (interval in } \mathrm{cm} \text { ) }\end{array}$ & $\begin{array}{c}\text { Sub-bottom } \\
\text { depth }(m)\end{array}$ & \multicolumn{2}{|c|}{$\begin{array}{l}\text { Sonic velocity } \\
(\mathrm{km} / \mathrm{s})\end{array}$} & $\begin{array}{c}\text { Acoustic } \\
\text { impedance } \\
\left(\mathrm{g} \cdot 10^{5} / \mathrm{cm}^{2} \cdot \mathrm{s}\right)\end{array}$ & $\begin{array}{l}\text { Unconfined } \\
\text { compression } \\
\text { strength } \\
(\mathrm{kPa})\end{array}$ & $\begin{array}{c}\text { Bulk } \\
\text { density } \\
\left(\mathrm{g} / \mathrm{cm}^{3}\right)\end{array}$ & $\begin{array}{l}\text { Grain } \\
\text { density } \\
\left(\mathrm{g} / \mathrm{cm}^{3}\right)\end{array}$ & $\begin{array}{c}\text { Porosity } \\
(\%)\end{array}$ & $\begin{array}{c}\text { Water } \\
\text { content } \\
(\%)\end{array}$ & $\begin{array}{c}\text { Carbonate } \\
\text { content } \\
(\%)\end{array}$ \\
\hline $21-4,75$ & 341.95 & 1.660 & 1.740 & 2.623 & & 1.58 & 2.30 & 58 & 64 & \\
\hline $21-5,75$ & 343.45 & 1.647 & 1.746 & 2.652 & & 1.61 & 2.32 & 57 & 60 & 44 \\
\hline $22-1,75$ & 347.05 & 1.880 & 1.922 & 3.628 & & 1.93 & 2.52 & 40 & 28 & \\
\hline $22-2,75$ & 348.55 & 1.825 & 1.898 & 3.340 & & 1.83 & 2.42 & 44 & 34 & 53 \\
\hline $22-3,75$ & 350.05 & 2.383 & 2.494 & 4.957 & & 2.08 & 2.51 & 29 & 18 & \\
\hline $22-4,75$ & 351.55 & 1.891 & 1.867 & 3.347 & & 1.77 & 2.40 & 47 & 39 & \\
\hline $22-5,75$ & 353.05 & 1.783 & 1.869 & 3.085 & & 1.73 & 2.39 & 50 & 44 & 35 \\
\hline $23-1,75$ & 356.65 & 1.775 & 1.901 & 3.213 & & 1.81 & 2.44 & 46 & 37 & \\
\hline $23-2,75$ & 358.15 & 1.762 & 1.828 & 3.154 & & 1.79 & 2.48 & 48 & 40 & 39 \\
\hline $23-3,75$ & 359.65 & 1.980 & 2.135 & 3.841 & & 1.94 & 2.47 & 38 & 26 & \\
\hline $23-4,75$ & 361.15 & 2.230 & 2.402 & 4.148 & & 1.86 & 2.38 & 39 & 29 & \\
\hline $23-5,75$ & 362.65 & 1.872 & 1.996 & 3.426 & & 1.83 & 2.45 & 45 & 33 & 31 \\
\hline $24-1,75$ & 366.25 & 1.947 & 2.091 & 3.738 & & 1.92 & 2.51 & 40 & 29 & \\
\hline $24-2,75$ & 367.75 & 1.878 & 1.851 & 3.456 & & 1.84 & 2.44 & 44 & 34 & 31 \\
\hline $24-3,75$ & 369.25 & 1.856 & 2.002 & 3.304 & & 1.78 & 2.41 & 47 & 39 & \\
\hline $24-4,75$ & 370.75 & 1.724 & 1.838 & 2.983 & & 1.73 & 2.38 & 49 & 43 & \\
\hline $24-5,75$ & 372.25 & 1.821 & 1.959 & 3.260 & & 1.79 & 2.38 & 45 & 36 & 38 \\
\hline $25-1,75$ & 375.85 & 1.890 & 2.005 & 3.572 & & 1.89 & 2.48 & 41 & 30 & \\
\hline $25-2,75$ & 377.35 & 1.737 & 1.810 & 3.074 & & 1.77 & 2.45 & 49 & 41 & 37 \\
\hline $25-3,75$ & 378.85 & 1.918 & 1.960 & 3.491 & & 1.82 & 2.50 & 47 & 38 & \\
\hline $25-4,75$ & 380.35 & 1.881 & 2.058 & 3.499 & & 1.86 & 2.45 & 43 & 32 & \\
\hline $25-5,75$ & 381.85 & 2.009 & 2.125 & 3.837 & & 1.91 & 2.45 & 39 & 27 & 41 \\
\hline $26-1,75$ & 385.45 & 2.018 & 2.181 & 3.996 & & 1.98 & 2.52 & 37 & 25 & 71 \\
\hline $26-2,80$ & 387.00 & 1.988 & 2.189 & 3.896 & & 1.96 & 2.47 & 36 & 25 & 38 \\
\hline $26-3,80$ & 388.50 & 1.821 & 1.978 & 3.369 & & 1.85 & 2.48 & 44 & 34 & \\
\hline $26-4,75$ & 389.95 & 2.082 & 2.334 & 4.289 & & 2.06 & 2.57 & 34 & 21 & \\
\hline $26-5,75$ & 391.45 & 1.884 & 2.115 & 3.467 & & 1.84 & 2.42 & 43 & 33 & 26 \\
\hline $27-1,75$ & 395.05 & 1.920 & 2.072 & 3.610 & & 1.88 & 2.51 & 44 & 33 & \\
\hline $27-2,75$ & 396.55 & 1.939 & 2.131 & 3.645 & & 1.88 & 2.44 & 41 & 30 & 32 \\
\hline $27-3,75$ & 398.05 & 1.968 & 2.157 & 3.759 & & 1.91 & 2.44 & 39 & 28 & \\
\hline $27-4,75$ & 399.55 & 1.853 & 2.026 & 3.428 & & 1.85 & 2.46 & 44 & 34 & \\
\hline $27-5,75$ & 401.05 & 2.000 & 2.159 & 3.820 & & 1.91 & 2.46 & 39 & 28 & 42 \\
\hline $28-1,75$ & 404.65 & 1.942 & 2.132 & 3.690 & & 1.90 & 2.47 & 40 & 29 & \\
\hline $28-2,75$ & 406.15 & 2.095 & 2.317 & 4.211 & & 2.01 & 2.50 & 34 & 22 & \\
\hline $28-3,75$ & 407.65 & 1.956 & 2.198 & 3.716 & & 1.90 & 2.43 & 39 & 27 & 30 \\
\hline $29-1,75$ & 414.25 & 1.968 & 2.210 & 3.720 & & 1.89 & 2.40 & 38 & 27 & \\
\hline $29-2,75$ & 415.75 & 2.050 & 2.268 & 4.080 & & 1.99 & 2.46 & 34 & 22 & 39 \\
\hline $29-3,75$ & 417.25 & 1.911 & 2.092 & 3.593 & & 1.88 & 2.49 & 42 & 31 & \\
\hline $29-4,75$ & 418.75 & 2.000 & 2.221 & 3.980 & & 1.99 & 2.47 & 34 & 22 & \\
\hline $29-5,75$ & 420.25 & 1.900 & 2.076 & 3.496 & & 1.84 & 2.42 & 42 & 32 & 23 \\
\hline $30-1,75$ & 423.85 & 1.994 & 2.234 & 3.888 & & 1.95 & 2.49 & 38 & 26 & \\
\hline $30-2,75$ & 425.35 & 1.999 & 2.215 & 3.918 & & 1.96 & 2.49 & 37 & 25 & 31 \\
\hline $30-3,75$ & 426.85 & 1.941 & 2.123 & 3.591 & & 1.85 & 2.44 & 43 & 33 & \\
\hline $30-4,75$ & 428.35 & 2.069 & 2.549 & 4.366 & & 2.11 & 2.57 & 30 & 18 & 52 \\
\hline $31-1,75$ & 433.45 & 2.258 & 2.572 & 4.855 & & 2.15 & 2.59 & 29 & 17 & \\
\hline $31-2,75$ & 434.95 & 2.217 & 2.483 & 4.700 & & 2.12 & 2.58 & 30 & 18 & 52 \\
\hline $31-3,75$ & 436.45 & 2.113 & 2.433 & 4.374 & & 2.07 & 2.57 & 33 & 20 & \\
\hline $31-4,75$ & 437.95 & 2.219 & 2.496 & 4.704 & & 2.12 & 2.58 & 30 & 18 & \\
\hline $31-5,75$ & 439.45 & 2.129 & 2.403 & 4.386 & & 2.06 & 2.56 & 33 & 21 & 45 \\
\hline $32-1,75$ & 443.05 & 2.070 & 2.259 & 4.285 & & 2.07 & 2.55 & 32 & 20 & \\
\hline $32-2,75$ & 444.55 & 2.206 & 2.510 & 4.677 & & 2.12 & 2.56 & 30 & 17 & 53 \\
\hline $32-3,75$ & 446.05 & 2.162 & 2.344 & 4.454 & & 2.06 & 2.54 & 32 & 20 & \\
\hline $32-4,75$ & 447.55 & 2.087 & 2.228 & 4.299 & & 2.06 & 2.57 & 34 & 21 & \\
\hline $32-5,75$ & 449.05 & 2.087 & 2.301 & 4.299 & & 2.06 & 2.58 & 35 & 22 & 45 \\
\hline $33-1,75$ & 452.65 & 2.197 & 2.403 & 4.746 & & 2.16 & 2.64 & 31 & 18 & \\
\hline $33-2,75$ & 454.15 & 2.108 & 2.290 & 4.342 & & 2.06 & 2.56 & 33 & 21 & 44 \\
\hline $33-3,75$ & 455.65 & 2.255 & 2.383 & 4.645 & & 2.06 & 2.52 & 32 & 19 & \\
\hline $33-4,75$ & 457.15 & 2.081 & 2.260 & 4.204 & & 2.02 & 2.52 & 35 & 22 & \\
\hline $33-5,75$ & 458.65 & 2.083 & 2.286 & 4.124 & & 1.98 & 2.44 & 34 & 22 & 43 \\
\hline $34-1,75$ & 462.25 & 2.139 & 2.241 & 4.385 & & 2.05 & 2.58 & 35 & 22 & \\
\hline $34-2,75$ & 463.75 & 2.034 & 2.217 & 4.170 & & 2.05 & 2.61 & 36 & 23 & 35 \\
\hline $34-3,75$ & 465.25 & 2.049 & 2.282 & 4.180 & & 2.04 & 2.59 & 36 & 23 & \\
\hline $34-4,75$ & 466.75 & 1.921 & 2.228 & 3.765 & & 1.96 & 2.67 & 44 & 31 & \\
\hline $34-5,75$ & 468.25 & 1.975 & 2.254 & 3.911 & & 1.98 & 2.55 & 39 & 26 & 33 \\
\hline $35-1,75$ & 471.85 & 2.019 & 2.144 & 3.876 & & 1.92 & 2.47 & 39 & 27 & \\
\hline $35-2,75$ & 473.35 & 1.889 & 2.150 & 3.721 & & 1.97 & 2.58 & 40 & 27 & 27 \\
\hline $35-4,75$ & 476.35 & 2.062 & 2.192 & 4.042 & & 1.96 & 2.58 & 40 & 28 & \\
\hline $35-5,75$ & 477.85 & 1.949 & 2.160 & 3.859 & & 1.98 & 2.58 & 40 & 27 & 31 \\
\hline $36-1,75$ & 481.45 & 2.045 & 2.231 & 4.070 & & 1.99 & 2.53 & 36 & 24 & \\
\hline $36-2,75$ & 482.95 & 2.108 & 2.258 & 4.279 & & 2.03 & 2.55 & 35 & 23 & 41 \\
\hline $36-3,75$ & 484.45 & 2.047 & 2.258 & 4.114 & & 2.01 & 2.52 & 35 & 23 & \\
\hline $37-1,75$ & 491.05 & 1.984 & 2.211 & 3.948 & & 1.99 & 2.51 & 36 & 23 & \\
\hline $37-2,75$ & 492.55 & 1.979 & 2.193 & $\begin{array}{l}4.037 \\
\end{array}$ & & 2.04 & 2.47 & 35 & 22 & 28 \\
\hline $37-3,75$ & 494.05 & 2.045 & 2.224 & 4.090 & & 2.00 & 2.54 & 36 & 24 & \\
\hline $37-4,75$ & 495.55 & 1.980 & 2.221 & 3.980 & & 2.01 & 2.63 & 39 & 26 & \\
\hline $37-5,75$ & 497.05 & 2.098 & 2.195 & 4.259 & & 2.03 & 2.53 & 34 & 22 & 36 \\
\hline
\end{tabular}


Table 5 (continued).

\begin{tabular}{|c|c|c|c|c|c|c|c|c|c|c|}
\hline $\begin{array}{l}\text { Core-Section } \\
\text { (interval in } \mathrm{cm} \text { ) }\end{array}$ & $\begin{array}{c}\text { Sub-bottom } \\
\text { depth }(\mathrm{m})\end{array}$ & \multicolumn{2}{|c|}{$\begin{array}{c}\text { Sonic velocity } \\
(\mathrm{km} / \mathrm{s})\end{array}$} & $\begin{array}{c}\text { Acoustic } \\
\text { impedance } \\
\left(\mathrm{g} \cdot 10^{5} / \mathrm{cm}^{2} \cdot \mathrm{s}\right)\end{array}$ & $\begin{array}{l}\text { Unconfined } \\
\text { compression } \\
\text { strength } \\
(\mathrm{kPa})\end{array}$ & $\begin{array}{c}\text { Bulk } \\
\text { density } \\
\left(\mathrm{g} / \mathrm{cm}^{3}\right)\end{array}$ & $\begin{array}{l}\text { Grain } \\
\text { density } \\
\left(\mathrm{g} / \mathrm{cm}^{3}\right)\end{array}$ & $\begin{array}{c}\text { Porosity } \\
(\%)\end{array}$ & $\begin{array}{c}\text { Water } \\
\text { content } \\
(\%)\end{array}$ & $\begin{array}{c}\text { Carbonate } \\
\text { content } \\
(\%)\end{array}$ \\
\hline $38-1,75$ & 500.65 & 2.076 & 2.277 & 4.380 & & 2.11 & 2.60 & 32 & 19 & \\
\hline $38-2,75$ & 502.15 & 2.052 & 2.203 & 4.125 & & 2.01 & 2.53 & 35 & 23 & 39 \\
\hline $38-3,75$ & 503.65 & 2.048 & 2.244 & 4.157 & & 2.03 & 2.56 & 36 & 23 & \\
\hline $38-4,75$ & 505.15 & 1.988 & 2.233 & 4.056 & & 2.04 & 2.58 & 35 & 23 & \\
\hline $38-5,75$ & 506.65 & 2.049 & 2.243 & 4.221 & & 2.06 & 2.59 & 35 & 22 & 38 \\
\hline $39-1,75$ & 510.25 & 1.913 & 2.201 & 3.845 & & 2.01 & 2.56 & 37 & 24 & \\
\hline $39-2,75$ & 511.75 & 2.007 & 2.011 & 4.074 & & 2.03 & 2.56 & 35 & 23 & 37 \\
\hline $39-3,75$ & 513.25 & 2.060 & 2.290 & 4.264 & & 2.07 & 2.58 & 34 & 21 & \\
\hline $39-4,75$ & 514.75 & 2.000 & 2.177 & 4.040 & & 2.02 & 2.54 & 36 & 23 & \\
\hline $39-5,75$ & 516.25 & 2.077 & 2.181 & 4.112 & & 1.98 & 2.49 & 35 & 23 & 35 \\
\hline $40-1,75$ & 519.85 & 2.029 & 2.265 & 4.159 & & 2.05 & 2.58 & 35 & 22 & \\
\hline $40-2,75$ & 521.35 & 2.033 & 2.303 & 4.249 & & 2.09 & 2.61 & 33 & 20 & 45 \\
\hline $40-3,75$ & 522.85 & 2.147 & 2.359 & 4.487 & & 2.09 & 2.56 & 31 & 19 & \\
\hline $40-4,75$ & 524.35 & 2.104 & 2.243 & 4.355 & & 2.07 & 2.61 & 35 & 22 & 41 \\
\hline $41-2,75$ & 530.95 & 2.031 & 2.252 & 4.103 & & 2.02 & 2.55 & 36 & 23 & 34 \\
\hline $41-4,75$ & 533.95 & 2.037 & 2.206 & 4.196 & & 2.06 & 2.57 & 34 & 21 & \\
\hline $41-4,75$ & 533.95 & 2.094 & 2.296 & 4.376 & & 2.09 & 2.60 & 33 & 20 & \\
\hline $41-5,75$ & 535.45 & 2.052 & 2.201 & 4.248 & & 2.07 & 2.59 & 34 & 21 & \\
\hline $41-6,75$ & 536.95 & 2.079 & 2.273 & $\begin{array}{l}4.304 \\
\end{array}$ & & 2.07 & 2.58 & 34 & 21 & \\
\hline $42-1,75$ & 539.05 & 1.996 & 2.220 & $\begin{array}{l}4.152 \\
4.152\end{array}$ & & 2.08 & 2.61 & 34 & 21 & \\
\hline $42-2,75$ & 540.55 & 2.043 & 2.297 & 4.290 & & 2.10 & 2.59 & 32 & 19 & 46 \\
\hline $42-3,75$ & 542.05 & 2.072 & 2.257 & 4.351 & & 2.10 & 2.60 & 33 & 20 & \\
\hline $42-4,78$ & 543.58 & 2.139 & 2.401 & 4.513 & & 2.11 & 2.66 & 34 & 21 & \\
\hline $42-5,75$ & 545.05 & 2.087 & 2.341 & 4.362 & & 2.09 & 2.62 & 34 & 21 & 43 \\
\hline $43-1,75$ & 548.65 & 2.023 & 2.143 & 4.167 & & 2.06 & 2.60 & 35 & 22 & \\
\hline $43-2,75$ & 550.15 & 2.314 & 2.430 & 4.859 & & 2.10 & 2.55 & 31 & 18 & 52 \\
\hline $43-3,75$ & 551.65 & 2.132 & 2.267 & 4.477 & & 2.10 & 2.66 & 35 & 22 & \\
\hline $43-4,75$ & 553.15 & 2.330 & 2.483 & 5.010 & & 2.15 & 2.60 & 29 & 17 & \\
\hline $43-5,75$ & 554.65 & 2.404 & 2.628 & 5.217 & & 2.17 & 2.60 & 28 & 16 & 66 \\
\hline $44-1,75$ & 558.25 & $\begin{array}{l}2.404 \\
2.410\end{array}$ & $\begin{array}{l}2.020 \\
2.464\end{array}$ & 5.157 & & 2.14 & 2.60 & 30 & 18 & \\
\hline $44-2,75$ & 559.75 & 2.586 & 2.663 & 5.560 & & 2.15 & 2.60 & 29 & 17 & 60 \\
\hline $44-3,75$ & 561.25 & 2.460 & 2.575 & 5.191 & & 2.11 & 2.57 & 31 & 18 & \\
\hline $44-4,75$ & 562.75 & 2.124 & 2.324 & 4.503 & & 2.12 & 2.60 & 32 & 19 & \\
\hline $44-5,75$ & 564.25 & 2.205 & 2.051 & 4.498 & & 2.04 & 2.60 & 36 & 23 & 40 \\
\hline $44-6,75$ & 565.75 & 1.967 & 2.142 & 3.973 & & 2.02 & 2.57 & 37 & 24 & \\
\hline $45-1,75$ & 567.85 & 1.950 & 2.087 & 3.978 & & 2.04 & 2.59 & 36 & 23 & \\
\hline $45 \cdot 2,75$ & 569.35 & 1.993 & 2.133 & 4.106 & & 2.06 & 2.58 & 34 & 22 & 35 \\
\hline $45-3,75$ & 570.85 & 1.958 & 2.177 & 4.014 & & 2.05 & 2.57 & 34 & 22 & \\
\hline $45-4,75$ & 572.35 & 2.004 & 2.142 & 4.128 & & 2.06 & 2.58 & 34 & 21 & 33 \\
\hline $45-5,75$ & 573.85 & 2.115 & 2.157 & 4.357 & & 2.06 & 2.55 & 33 & 20 & \\
\hline $46-1,75$ & 577.45 & 2.201 & 2.418 & 4.556 & & 2.07 & 2.58 & 34 & 21 & \\
\hline $46-2,75$ & 578.95 & 1.926 & 2.100 & 3.910 & & 2.03 & 2.59 & 37 & 24 & 28 \\
\hline $46-3,75$ & 580.45 & 2.036 & 2.012 & 4.153 & & 2.04 & 2.60 & 36 & 23 & \\
\hline $46-4,75$ & 581.95 & 2.062 & 2.053 & 4.186 & & 2.03 & 2.59 & 37 & 24 & 27 \\
\hline $47-1,75$ & 587.05 & 1.893 & 2.061 & 3.862 & & 2.04 & 2.60 & 37 & 24 & \\
\hline $47-2,75$ & 588.55 & 1.873 & 2.083 & 3.783 & & 2.02 & 2.63 & 37 & 26 & 17 \\
\hline $47-3,75$ & 590.05 & 1.923 & 2.085 & 3.846 & & 2.00 & 2.58 & 38 & 25 & \\
\hline $47-4,75$ & 591.55 & 1.923 & 2.105 & 3.865 & & 2.01 & 2.60 & 38 & 25 & \\
\hline $47-5,75$ & 593.05 & 1.901 & 2.117 & 3.802 & & 2.00 & 2.57 & 38 & 25 & 25 \\
\hline $48-1,75$ & 596.65 & 1.879 & 2.077 & 3.664 & & 1.95 & 2.47 & 36 & 25 & \\
\hline $48-2,75$ & 598.15 & 1.914 & 2.580 & 3.809 & & 1.99 & 2.55 & 38 & 25 & 18 \\
\hline $48-3,75$ & 599.65 & $\begin{array}{l}1.998 \\
\end{array}$ & 2.230 & 3.956 & & 1.98 & 2.54 & 38 & 26 & \\
\hline $48-4,75$ & 601.15 & 2.191 & & 4.513 & & 2.06 & 2.62 & 36 & 23 & 22 \\
\hline $49-1,75$ & 606.25 & 1.903 & 2.040 & 3.692 & & 1.94 & 2.57 & 41 & 29 & 18 \\
\hline $49-2,75$ & 607.75 & 1.926 & 2.036 & 3.736 & & 1.94 & 2.57 & 42 & 30 & \\
\hline $49-3,75$ & 609.25 & 2.196 & 2.367 & 4.546 & & 2.07 & 2.57 & 33 & 20 & \\
\hline $49-4,75$ & 610.75 & 1.963 & 2.042 & 3.847 & & 1.96 & 2.45 & 35 & 24 & \\
\hline $49-5,75$ & 612.25 & 2.065 & 2.270 & 4.233 & & 2.05 & 2.56 & 34 & 22 & 42 \\
\hline $50-1,75$ & 615.85 & 2.058 & 2.148 & 4.095 & & 1.99 & 2.51 & 36 & 24 & 29 \\
\hline $50-2,75$ & 617.35 & 2.033 & 2.122 & 4.046 & & 1.99 & 2.49 & 35 & 23 & \\
\hline $50-3,75$ & 618.85 & 1.915 & 2.162 & 3.868 & & 2.02 & 2.53 & 35 & 23 & \\
\hline $50-4,75$ & 620.35 & 2.076 & 2.226 & 4.297 & & 2.07 & 2.58 & 33 & 21 & \\
\hline $50-5,75$ & 621.85 & 2.081 & 2.261 & 4.266 & & 2.05 & 2.53 & 33 & 21 & 41 \\
\hline $51-1,75$ & 625.45 & 1.973 & 2.172 & 3.867 & & 1.96 & 2.51 & 38 & 26 & \\
\hline $51-2,75$ & 626.95 & 2.031 & 2.217 & 4.123 & & 2.03 & 2.54 & 35 & 22 & 41 \\
\hline $51-3,75$ & 628.45 & 1.966 & 2.177 & 3.991 & & 2.03 & 2.63 & 38 & 25 & \\
\hline $52-1,75$ & 635.05 & 2.061 & 2.265 & 4.246 & & 2.06 & 2.50 & 31 & 19 & \\
\hline $52-2,75$ & 636.55 & 2.070 & 2.267 & 4.202 & & 2.03 & 2.54 & 34 & 22 & 43 \\
\hline $52-3,75$ & 638.05 & 2.054 & 2.207 & 4.170 & & 2.03 & 2.53 & 34 & 21 & \\
\hline $52-4,75$ & 639.55 & 2.134 & 2.358 & 4.481 & & 2.10 & 2.55 & 30 & 18 & \\
\hline $52-5,75$ & 641.05 & 2.089 & 2.278 & 4.366 & & 2.09 & 2.55 & 31 & 19 & 47 \\
\hline $53-1,75$ & 644.65 & 1.915 & 2.146 & 3.811 & & 1.99 & 2.58 & 39 & 26 & 9 \\
\hline $54-1,75$ & 649.15 & 2.085 & 2.282 & 4.379 & & 2.10 & 2.62 & 33 & 20 & 40 \\
\hline $55-1,75$ & 654.25 & 2.079 & 2.194 & 4.324 & & 2.08 & 2.55 & 32 & 20 & 40 \\
\hline $55-2,75$ & 655.75 & 2.000 & 2.219 & 6.000 & & 3.00 & 2.58 & 27 & 9 & 40 \\
\hline $55-3,75$ & 657.25 & 2.081 & 2.241 & 4.266 & & 2.05 & 2.56 & 34 & 21 & \\
\hline
\end{tabular}


Table 5 (continued).

\begin{tabular}{|c|c|c|c|c|c|c|c|c|c|c|}
\hline $\begin{array}{l}\text { Core-Section } \\
\text { (interval in } \mathrm{cm} \text { ) }\end{array}$ & $\begin{array}{l}\text { Sub-bottom } \\
\text { depth }(\mathrm{m})\end{array}$ & \multicolumn{2}{|c|}{$\begin{array}{l}\text { Sonic velocity } \\
(\mathrm{km} / \mathrm{s})\end{array}$} & $\begin{array}{c}\text { Acoustic } \\
\text { impedance } \\
\left(\mathrm{g} \cdot 10^{5} / \mathrm{cm}^{2} \cdot \mathrm{s}\right)\end{array}$ & $\begin{array}{l}\text { Unconfined } \\
\text { compression } \\
\text { strength } \\
(\mathrm{kPa})\end{array}$ & $\begin{array}{c}\text { Bulk } \\
\text { density } \\
\left(\mathrm{g} / \mathrm{cm}^{3}\right)\end{array}$ & $\begin{array}{l}\text { Grain } \\
\text { density } \\
\left(\mathrm{g} / \mathrm{cm}^{3}\right)\end{array}$ & $\begin{array}{c}\text { Porosity } \\
(\%)\end{array}$ & $\begin{array}{c}\text { Water } \\
\text { content } \\
(\%)\end{array}$ & $\begin{array}{c}\text { Carbonate } \\
\text { content } \\
(\%)\end{array}$ \\
\hline $55-4,75$ & 658.75 & 2.083 & 2.279 & 4.374 & & 2.10 & 2.59 & 34 & 19 & \\
\hline $55-5,75$ & 660.25 & 2.174 & 2.313 & 4.478 & & 2.06 & 2.54 & 32 & 20 & 41 \\
\hline $56-1,75$ & 663.85 & 2.150 & 2.309 & 4.558 & & 2.12 & 2.58 & 30 & 18 & 45 \\
\hline $56-2,75$ & 665.35 & 2.107 & 2.320 & 4.383 & & 2.08 & 2.57 & 32 & 20 & \\
\hline $56-3,75$ & 666.85 & 2.170 & 2.282 & 4.514 & & 2.08 & 2.54 & 31 & 19 & \\
\hline $56-4,75$ & 668.35 & 2.031 & 2.245 & 4.143 & & 2.04 & 2.56 & 35 & 22 & \\
\hline $56-5,75$ & 669.85 & 2.325 & 2.519 & 4.929 & & 2.12 & 2.55 & 29 & 17 & 56 \\
\hline $57-1,75$ & 673.45 & 1.990 & 2.236 & 4.000 & & 2.01 & 2.49 & 34 & 22 & \\
\hline $57-2,75$ & 674.95 & 2.067 & 2.310 & 4.279 & & 2.07 & 2.57 & 33 & 20 & 37 \\
\hline $57-3,75$ & 676.45 & 1.904 & 2.192 & 3.789 & & 1.99 & 2.55 & 37 & 25 & \\
\hline $57-4,75$ & 677.95 & 2.143 & 2.095 & 4.522 & & 2.11 & 2.60 & 32 & 19 & \\
\hline $57-5,75$ & 679.45 & 2.274 & 2.266 & 4.730 & & 2.08 & 2.56 & 32 & 20 & 39 \\
\hline $59-1,75$ & 692.65 & 1.882 & 2.360 & 3.896 & & 2.07 & 2.56 & 33 & 20 & \\
\hline $59-2,75$ & 694.15 & 2.040 & 2.145 & 4.223 & & 2.07 & 2.56 & 33 & 20 & 41 \\
\hline $59-3,75$ & 695.65 & 1.959 & 2.229 & 4.036 & & 2.06 & 2.50 & 34 & 22 & \\
\hline $59.4,75$ & 697.15 & 1.943 & 2.253 & 3.789 & & 1.95 & 2.50 & 38 & 26 & \\
\hline $59-5,75$ & 698.65 & 2.100 & 2.230 & 4.305 & & 2.05 & 2.52 & 32 & 20 & 42 \\
\hline $60-1,75$ & 702.25 & 2.193 & 2.254 & 4.649 & & 2.12 & 2.62 & 32 & 19 & \\
\hline $60-2,75$ & 703.75 & 2.064 & 2.344 & & & & & & & 30 \\
\hline $60-3,75$ & 705.25 & 2.092 & 2.239 & 4.268 & & 2.04 & 2.53 & 33 & 21 & \\
\hline $60-4,75$ & 706.75 & 2.164 & 2.327 & 4.436 & & 2.05 & 2.51 & 32 & 20 & \\
\hline $60-5,75$ & 708.25 & 2.149 & 2.323 & 4.491 & & 2.09 & 2.62 & 34 & 21 & 41 \\
\hline $61-1,75$ & 711.85 & 2.070 & 2.210 & 4.140 & & 2.00 & 2.55 & 37 & 25 & \\
\hline $61-2,75$ & 713.35 & 2.050 & 2.249 & 4.141 & & 2.02 & 2.55 & 36 & 23 & 31 \\
\hline $61-3,75$ & 714.85 & 2.054 & 2.236 & 4.046 & & 1.97 & 2.51 & 37 & 25 & \\
\hline $61-4,75$ & 716.35 & 2.116 & 2.201 & 3.915 & & 1.85 & 2.36 & 39 & 29 & \\
\hline $61-5,75$ & 717.85 & 2.174 & 2.281 & 4.283 & & 1.97 & 2.50 & 37 & 25 & 32 \\
\hline $62-1,75$ & 721.45 & 2.059 & 2.217 & 4.077 & & 1.98 & 2.51 & 37 & 25 & \\
\hline $62-2,75$ & 722.95 & 2.139 & 2.376 & 4.406 & & 2.06 & 2.65 & 38 & 24 & 36 \\
\hline $62-3,75$ & 724.45 & 2.835 & 2.477 & & & & & & & \\
\hline $62-4,75$ & 725.95 & 2.174 & 2.161 & 4.457 & & 2.05 & 2.60 & 36 & 23 & \\
\hline $62-5,75$ & 727.45 & 2.187 & 2.343 & 4.265 & & 1.95 & 2.52 & 39 & 27 & 38 \\
\hline $63-1,75$ & 731.05 & 2.026 & 2.188 & 4.072 & & 2.01 & 2.55 & 36 & 24 & \\
\hline $63-2,75$ & 732.55 & 2.070 & 2.231 & 3.995 & & 1.93 & 2.54 & 41 & 29 & 26 \\
\hline $63-3,75$ & 734.05 & 2.060 & 2.286 & 4.017 & & 1.95 & 2.52 & 39 & 27 & \\
\hline $63-4,75$ & 735.55 & 2.115 & 2.199 & 4.040 & & 1.91 & 2.47 & 40 & 28 & \\
\hline $63-5,75$ & 737.05 & 2.150 & 2.239 & 4.085 & & 1.90 & 2.45 & 40 & 28 & 25 \\
\hline $64-1,75$ & 740.65 & 2.653 & 2.781 & 5.837 & & 2.20 & 2.65 & 28 & 16 & \\
\hline $64-2,75$ & 742.15 & 2.759 & 2.829 & 6.042 & & 2.19 & 2.29 & 8 & 4 & 47 \\
\hline $64-3,75$ & 743.65 & 2.176 & 2.443 & 4.635 & & 2.13 & 2.69 & 34 & 21 & \\
\hline $64-4,75$ & 745.15 & 2.125 & 2.379 & 4.420 & & 2.08 & 2.66 & 36 & 22 & \\
\hline $64-5,75$ & 746.65 & 1.998 & 2.138 & 4.056 & & 2.03 & 2.61 & 38 & 24 & 18 \\
\hline $65-1,75$ & 750.25 & 2.041 & 2.290 & 4.102 & & 2.01 & 2.63 & 36 & 26 & \\
\hline $65-2,71$ & 751.71 & 2.060 & 2.418 & 4.223 & & 2.05 & 2.64 & 37 & 24 & 12 \\
\hline $66-2,72$ & 761.32 & 2.008 & 1.971 & 4.157 & & 2.07 & 2.66 & 37 & 24 & 41 \\
\hline $66-3,72$ & 762.82 & 2.071 & 2.185 & 4.246 & & 2.05 & 2.66 & 38 & 24 & \\
\hline $66-4,72$ & 764.32 & 2.141 & 2.263 & 4.625 & & 2.16 & 2.67 & 31 & 18 & \\
\hline $67-1,75$ & 769.45 & 2.455 & 2.601 & 5.548 & & 2.26 & 2.68 & 26 & 14 & \\
\hline $67-2,46$ & 770.66 & 2.237 & 2.266 & 4.832 & & 2.16 & 2.65 & 31 & 18 & 62 \\
\hline $67-3,75$ & 772.45 & 2.410 & 2.179 & 5.085 & & 2.11 & 2.58 & 31 & 18 & \\
\hline $67-4,75$ & 773.95 & 2.215 & 2.236 & 4.474 & & 2.02 & 2.49 & 33 & 21 & \\
\hline $68-1,75$ & 779.05 & 2.265 & 2.377 & 4.938 & & 2.18 & 2.65 & 30 & 17 & \\
\hline $68-2,24$ & 780.04 & 2.283 & 2.304 & 5.000 & & 2.19 & 2.77 & 34 & 20 & 59 \\
\hline $68-3,46$ & 781.76 & 2.356 & 2.416 & 5.230 & & 2.22 & 2.70 & 29 & 16 & \\
\hline $68-4,76$ & 783.56 & 2.385 & 2.523 & 5.223 & & 2.19 & 2.67 & 30 & 17 & \\
\hline $68-5,75$ & 785.05 & 2.466 & 2.582 & 5.450 & & 2.21 & 2.67 & 28 & 16 & 64 \\
\hline $69-1,75$ & 788.65 & 2.735 & 2.804 & 6.291 & & 2.30 & 2.69 & 24 & 12 & \\
\hline $69-2,75$ & 790.15 & 2.810 & 3.073 & 6.547 & & 2.33 & 2.68 & 22 & 11 & 69 \\
\hline $69-3,75$ & 791.65 & 2.552 & 2.558 & 5.665 & & 2.22 & 2.68 & 28 & 16 & \\
\hline $69-4,75$ & 793.15 & 2.649 & 2.774 & 6.013 & & 2.27 & 2.69 & 26 & 14 & \\
\hline $69-5,75$ & 794.65 & 2.304 & 2.445 & 4.931 & & 2.14 & 2.67 & 33 & 20 & 57 \\
\hline $70-1,75$ & 798.25 & 2.307 & 2.452 & 5.006 & & 2.17 & 2.67 & 31 & 18 & \\
\hline $70-2,75$ & 799.75 & 2.324 & 2.422 & 5.043 & & 2.17 & 2.69 & 32 & 18 & 59 \\
\hline $70-3,75$ & 801.25 & 2.424 & 2.550 & 5.309 & & 2.19 & 2.70 & 31 & 18 & \\
\hline $70-4,75$ & 802.75 & 2.198 & 2.354 & 4.638 & & 2.11 & 2.66 & 34 & 21 & \\
\hline $70-5,75$ & 804.25 & 2.333 & 2.465 & 5.016 & & 2.15 & 2.69 & 33 & 19 & 57 \\
\hline $71-1,75$ & 807.85 & 2.203 & 2.299 & 4.582 & & 2.08 & 2.66 & 37 & 23 & \\
\hline $71-2,75$ & 809.35 & 2.323 & 2.458 & 5.064 & & 2.18 & 2.66 & 30 & 17 & 59 \\
\hline $71-3,75$ & 810.85 & 2.588 & 2.678 & 5.978 & & 2.31 & 2.71 & 24 & 12 & \\
\hline $71-4,75$ & 812.35 & 2.224 & 2.384 & 4.648 & & 2.09 & 2.60 & 33 & 20 & \\
\hline $71-5,75$ & 813.85 & 2.243 & 2.334 & 4.890 & & 2.18 & 2.65 & 30 & 17 & 60 \\
\hline
\end{tabular}

